Supplementary Information

for

\title{
Condensation of Macrocyclic Polyketides Produced by Penicillium sp. DRF2 with Mercaptopyruvate Represents a New Fungal Detoxification Pathway
}

Marcos V. de Castro, Laura P. Ióca, David E. Williams, Bruna Z. Costa, Carolina M. Mizuno, Mario F. C. Santos, Karen de Jesus, Éverton L. F. Ferreira, Mirna H. R. Seleghim, Lara D. Sette, Edenir R. Pereira Filho, Antonio G. Ferreira, Natália S. Gonçalves, Raquel A. Santos, Brian Patrick, Raymond J. Andersen, Roberto G. S. Berlinck*

\section{X-Ray Diffraction Analysis Data for Cyclothiocurvularin A (6) and the Dimethoxymethyl ester of Cyclothiocurvularin B (9)}

Page S3 - 1. Data Collection for Cyclothiocurvularin A (6) X-ray analysis.

Page S5 - Figure S1. Cyclothiocurvularin A molecule 1 in the asymmetric unit.

Page S5 - Figure S2. Cyclothiocurvularin A Molecule 2 in the asymmetric unit.

Page S7 - 1.1 Experimental details of Cyclothiocurvularin A (6) X-Ray diffraction analysis

Page S9 - Table S1. Fractional Atomic Coordinates $\left(\times 10^{4}\right)$ and Equivalent Isotropic Displacement Parameters

$\left(\AA^{2} \times 10^{3}\right)$ for cyclothiocurvularin A. $\mathrm{U}_{\text {eq }}$ is defined as $1 / 3$ of of the trace of the orthogonalised $\mathrm{U}_{\mathrm{IJ}}$ tensor.

Page S12 - Table S2. Anisotropic Displacement Parameters $\left(\AA^{2} \times 10^{3}\right)$ for cyclothiocurvularin A. The Anisotropic displacement factor exponent takes the form: $-2 \pi^{2}\left[h^{2} a^{* 2} U_{11}+2 h k a * b * U_{12}+\ldots\right]$.

Page S15 - Table S3. Bond Lengths for cyclothiocurvularin A.

Page S17 - Table S4. Bond Angles for cyclothiocurvularin A.

Page S19 - Table S5. Hydrogen Bonds for cyclothiocurvularin A.

Page S20 - Table S6. Torsion Angles for cyclothiocurvularin A.

Page S23 - Table S7. Hydrogen Atom Coordinates $\left(\AA \times 10^{4}\right)$ and Isotropic Displacement Parameters $\left(\AA^{2} \times 10^{3}\right)$ for cyclothiocurvularin A.

Page S27 - Table S8. Atomic Occupancy for cyclothiocurvularin A.

Page S29 - 2. Data Collection for dimethoxymethyl ester of cyclothiocurvularin B (9) X-ray analysis.

Page S31 - Figure S3. ORTEP-style picture of the molecule A of dimethoxymethyl ester of cyclothiocurvularin B (9).

Page S32 - Figure S4. ORTEP-style picture of the molecule B of dimethoxymethyl ester of cyclothiocurvularin B (9).

Page S33 - Figure S5. ORTEP-style image of both molecules in the asymmetric unit. 
Page S34 - 2.1 Experimental details of dimethoxymethyl ester of cyclothiocurvularin B (9) X-Ray diffraction analysis

Page S36 - Table S9. Fractional Atomic Coordinates $\left(\times 10^{4}\right)$ and Equivalent Isotropic Displacement Parameters $\left(\AA^{2} \times 10^{3}\right)$ for dimethoxymethyl ester of cyclothiocurvularin B (9). $\mathrm{U}_{\text {eq }}$ is defined as $1 / 3$ of of the trace of the orthogonalised $\mathrm{U}_{\mathrm{IJ}}$ tensor.

Page S39 - Table S10. Anisotropic Displacement Parameters $\left(\AA^{2} \times 10^{3}\right)$ for dimethoxymethyl ester 9. The Anisotropic displacement factor exponent takes the form: $-2 \pi^{2}\left[h^{2} a^{* 2} U_{11}+2 h k a * b * U_{12}+\ldots\right]$.

Page S42 - Table S11. Bond Lengths for dimethoxymethyl ester of cyclothiocurvularin B (9).

Page S43 - Table S12. Bond Angles for dimethoxymethyl ester of cyclothiocurvularin B (9).

Page S45 - Table S13. Hydrogen Bonds for dimethoxymethyl ester of cyclothiocurvularin B (9)

Page S46 - Table S14. Torsion Angles for dimethoxymethyl ester of cyclothiocurvularin B (9).

Page S49 - Table S15. Hydrogen Atom Coordinates $\left(\AA \times 10^{4}\right)$ and Isotropic Displacement Parameters $\left(\AA^{2} \times 10^{3}\right)$ for dimethoxymethyl ester of cyclothiocurvularin B (9). 


\section{Data Collection for Cyclothiocurvularin A (6) X-ray analysis}

A colourless block crystal of $2\left[\mathrm{C}_{19} \mathrm{H}_{22} \mathrm{O}_{8} \mathrm{~S}\right] .5 \mathrm{H}_{2} \mathrm{O} \cdot \mathrm{MeOH}$ having approximate dimensions of 0.10 x 0.12 x $0.35 \mathrm{~mm}$ was mounted on a glass fiber. All measurements were made on a Bruker X8 APEX II diffractometer with cross-coupled multilayer optics Mo-K $\alpha$ radiation. The data were collected at a temperature of $-173.0 \pm 2{ }^{\circ} \mathrm{C}$ to a maximum $2 \theta$ value of $50.8^{\circ}$. Data were collected in a series of $\phi$ and $\omega$ scans in $1.00^{\circ}$ oscillations with 20.0 -second exposures. The crystal-to-detector distance was $39.80 \mathrm{~mm}$.

\section{Data Reduction}

Of the 30225 reflections that were collected, 8055 were unique $\left(\mathrm{R}_{\text {int }}=0.068\right)$; equivalent reflections, excluding Friedel pairs, were merged. Data were collected and integrated using the Bruker $\mathrm{SAINT}^{1}$ software package. The linear absorption coefficient, $\mu$, for Mo-K $\alpha$ radiation is $2.06 \mathrm{~cm}^{-1}$. Data were corrected for absorption effects using the multi-scan technique $\left(\mathrm{SADABS}^{2}\right)$, with minimum and maximum transmission coefficients of 0.924 and 0.980 , respectively. The data were corrected for Lorentz and polarization effects.

\section{$\underline{\text { Structure Solution and Refinement }}$}

The structure cyclothiocurvularin A (6) was solved by direct methods. ${ }^{3}$ Cyclothiocurvularin A (6) crystallizes with two crystallographically independent molecules in the asymmetric unit (Figs. 1 and 2). The two molecules have considerably different geometries (Fig. 3). The second molecule is also slightly disordered. Mild restraints were employed to maintain similar bond lengths. Finally, the asymmetric unit contains a large amount of recrystallization solvent. There are approximately 5 molecules of water, and one molecule of $\mathrm{MeOH}$ distributed over 10 sites. All non-hydrogen atoms were refined anisotropically, however disordered atoms from different fragments occupying roughly the same space were given equivalent anisotropic displacement parameters. All hydrogen atoms were placed in calculated positions. The absolute configurations for the chiral centres on each molecule (R, R, S, and S for C10/29, C11/30, $\mathrm{C} 15 / 34$, and $\mathrm{C} 18 / 37$, respectively) were determined on the basis of the refined Flack parameter, ${ }^{12} 0.05(5)$. The final cycle of full-matrix least-squares refinement ${ }^{4}$ on $\mathrm{F}^{2}$ was based on 8055 reflections and 662 variable parameters and converged (largest parameter shift was 0.00 times its esd) with unweighted and weighted agreement factors of:

$$
\begin{gathered}
\mathrm{R} 1(\mathrm{I}>2.00 \sigma(\mathrm{I}))=\Sigma\|\mathrm{Fo}|-| \mathrm{Fc}\| / \Sigma|\mathrm{Fo}|=0.041 \\
\mathrm{wR} 2(\text { all data })=\left[\Sigma\left(\mathrm{w}\left(\mathrm{Fo}^{2}-\mathrm{Fc}^{2}\right)^{2}\right) / \Sigma \mathrm{w}\left(\mathrm{Fo}^{2}\right)^{2}\right]^{1 / 2}=0.106
\end{gathered}
$$


The standard deviation of an observation of unit weight ${ }^{5}$ was 1.02. The weighting scheme was based on counting statistics. The maximum and minimum peaks on the final difference Fourier map corresponded to 0.29 and $-0.28 \mathrm{e}^{-} / \AA^{3}$, respectively.

Neutral atom scattering factors were taken from Cromer and Waber. ${ }^{6}$ Anomalous dispersion effects were included in Fcalc; ${ }^{7}$ the values for $\Delta \mathrm{f}^{\prime}$ and $\Delta \mathrm{f}^{\prime \prime}$ were those of Creagh and McAuley. ${ }^{8}$ The values for the mass attenuation coefficients are those of Creagh and Hubbell. ${ }^{9}$ All refinements were performed using the SHELXL-2014 ${ }^{10}$ via the Olex $2^{11}$ interface.

\section{References}

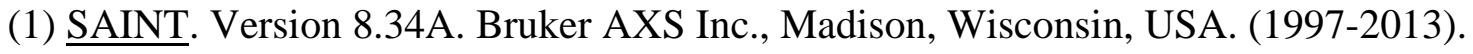

(2) SADABS 2014/5 - Krause, L., Herbst-Irmer, R., Sheldrick, G. M. \& Stalke, D. J. Appl. Crystallogr. 2015, 48, 3-10.

(3) SHELXT: Sheldrick, G. M.; Acta Cryst., 2015, A71, 3-8.

(4) Least Squares function minimized:

$$
\sum w\left(\mathrm{~F}_{\mathrm{O}}^{2}-\mathrm{F}_{\mathrm{c}}{ }^{2}\right)^{2}
$$

(5) Standard deviation of an observation of unit weight:

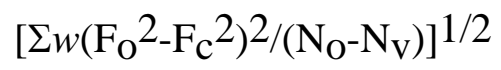

where: $\quad \mathrm{N}_{\mathrm{O}}=$ number of observations; $\quad \mathrm{N}_{\mathrm{V}}=$ number of variables

(6) Cromer, D. T. \& Waber, J. T.; "International Tables for X-ray Crystallography", Vol. IV, The Kynoch Press, Birmingham, England, Table 2.2 A (1974).

(7) Ibers, J. A. \& Hamilton, W. C.; Acta Cryst. 1964, 17, 781.

(8) Creagh, D. C. \& McAuley, W.J .; "International Tables for Crystallography", Vol C, (A.J.C. Wilson, ed.), Kluwer Academic Publishers, Boston, Table 4.2.6.8, pages 219-222 (1992).

(9) Creagh, D. C. \& Hubbell, J.H..; "International Tables for Crystallography", Vol C, (A.J.C. Wilson, ed.), Kluwer Academic Publishers, Boston, Table 4.2.4.3, pages 200-206 (1992).

(10) SHELXL-2014 - Sheldrick, G. M.; Acta Cryst. 2015, A71, 3-8.

(11) OLEX2 - V1.2.7 - Dolomanov, O.V.; Bourhis, L.J.; Gildea, R.J.; Howard, J.A.K.; Puschmann, H., OLEX2: A complete structure solution, refinement and analysis program. J. Appl. Cryst. 2009, 42, 339341.

(12) Parsons, S.; Flack, H. D.; Wagner, T. Acta Cryst. 2013, B69, 249-259. 


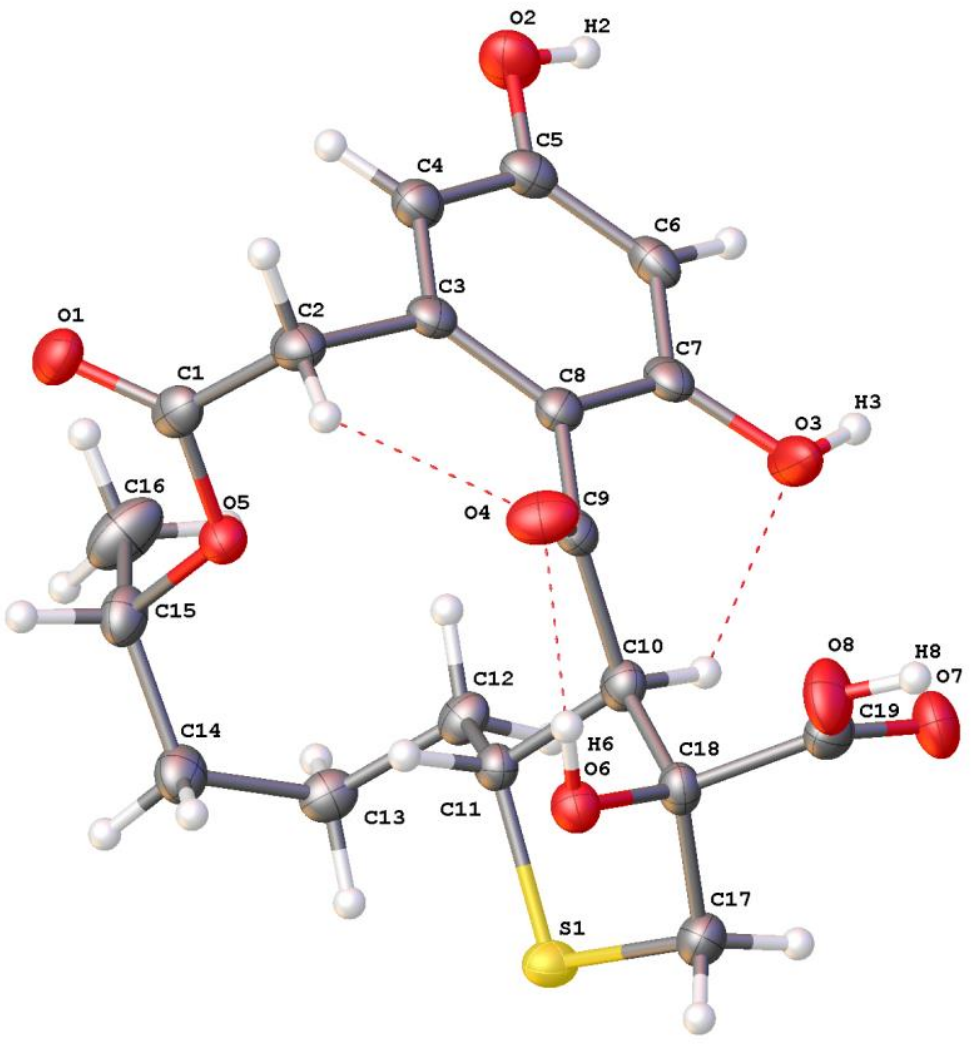

Figure S1. Cyclothiocurvularin A molecule 1 in the asymmetric unit.

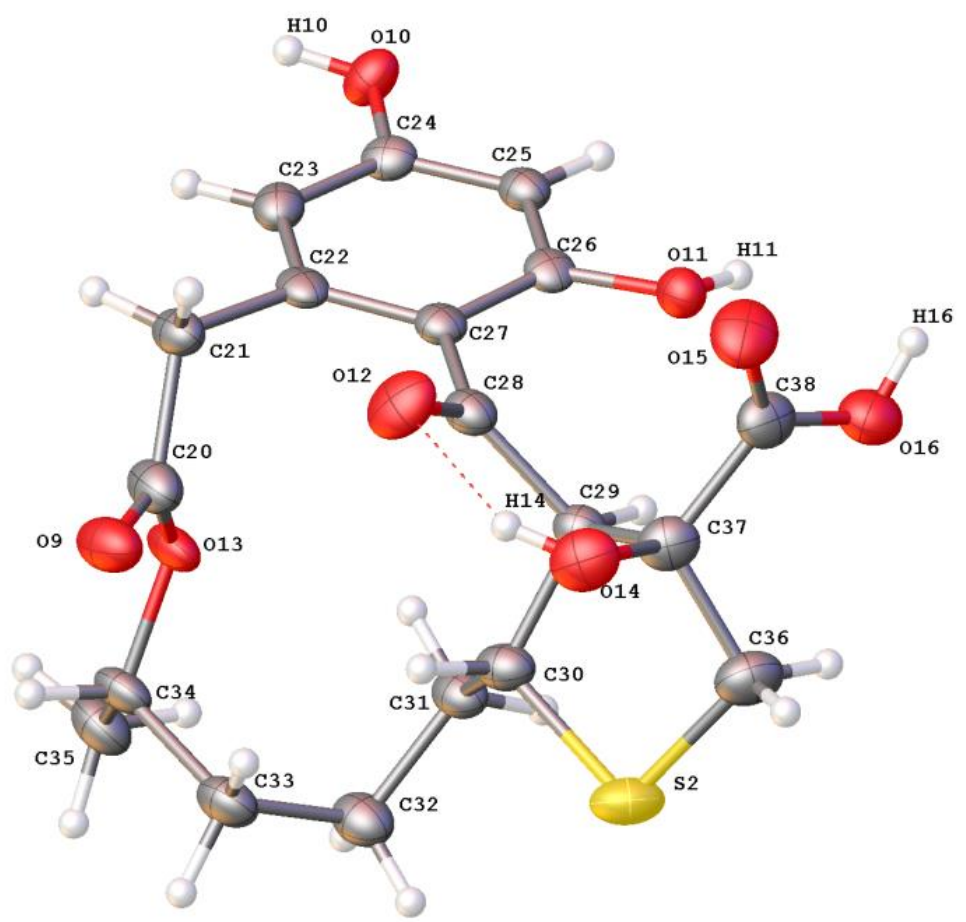

Figure S2. Cyclothiocurvularin A Molecule 2 in the asymmetric unit. 


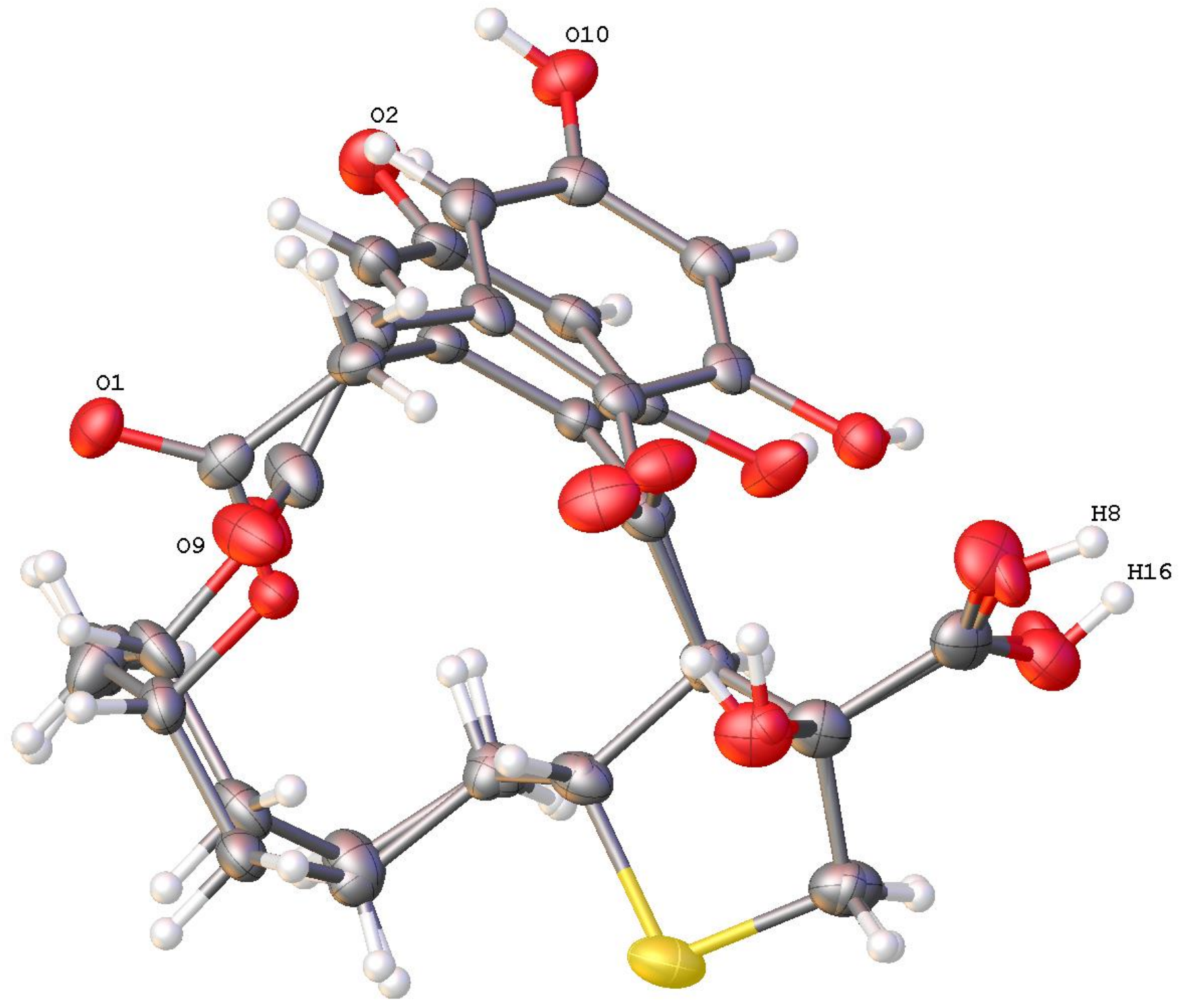




\subsection{Experimental details of Cyclothiocurvularin A (6) X-Ray diffraction analysis}

A. Cyclothiocurvularin A (6) Crystal Data

Empirical Formula

Formula Weight

Crystal Colour, Habit

Crystal Dimensions

Crystal System

Lattice Type

Lattice Parameters

Space Group

$\mathrm{Z}$ value

Dcalc

F000

$\mu(\operatorname{MoK} \alpha)$

B. Intensity Measurements

Diffractometer

Radiation

Data Images
$\mathrm{C}_{39} \mathrm{H}_{55} \mathrm{O}_{22} \mathrm{~S}_{2}$

942.66

colourless, block

$0.10 \times 0.12 \times 0.35 \mathrm{~mm}$

orthorhombic

primitive

$a=10.7952(7) \AA$

$\mathrm{b}=17.2819(11) \AA$

$c=23.5916(13) \AA$

$\alpha=90^{\circ}$

$\beta=90^{\circ}$

$\gamma=90^{\circ}$

$\mathrm{V}=4401.3(5) \AA^{3}$

$P 22_{12}{ }_{1} \quad$ (\#19)

4

$1.483 \mathrm{~g} / \mathrm{cm}^{3}$

1999.00

$2.06 \mathrm{~cm}^{-1}$

$\operatorname{MoK} \alpha(\lambda=0.71073 \AA)$

graphite monochromated

677 exposures@ 20.0 seconds 
Detector Position

$2 \theta_{\max }$

No. of Reflections Measured

Corrections

C. Structure Solution and Refinement

Structure Solution

Refinement

Function Minimized

Least Squares Weights

Anomalous Dispersion

No. Observations $(\mathrm{I}>0.00 \sigma(\mathrm{I}))$

No. Variables

Reflection/Parameter Ratio

Residuals (refined on $\mathrm{F}^{2}$, all data): R1; wR2

Goodness of Fit Indicator

No. Observations $(\mathrm{I}>2.00 \sigma(\mathrm{I}))$

Residuals (refined on $\mathrm{F}^{2}$ ): R1; wR2

Max Shift/Error in Final Cycle

Maximum peak in Final Diff. Map

Minimum peak in Final Diff. Map
$39.80 \mathrm{~mm}$

$50.8^{\circ}$

Total: 30225

Unique: $8055\left(\mathrm{R}_{\text {int }}=0.068\right)$

Absorption $\left(\mathrm{T}_{\min }=0.924, \mathrm{~T}_{\max }=0.980\right)$

Lorentz-polarization

Direct Methods (XT)

Full-matrix least-squares on $\mathrm{F}^{2}$

$\Sigma \mathrm{w}\left(\mathrm{Fo}^{2}-\mathrm{Fc}^{2}\right)^{2}$

$\mathrm{w}=1 /\left(\sigma^{2}\left(\mathrm{Fo}^{2}\right)+(0.0534 \mathrm{P})^{2}+0.0000 \mathrm{P}\right)$

All non-hydrogen atoms

8055

662

12.17

$0.056 ; 0.106$

1.02

6376

$0.041 ; 0.094$

0.00

$0.29 \mathrm{e}^{-/ \AA} 3$

$-0.28 \mathrm{e}^{-} / \AA^{3}$ 
Table S1. Fractional Atomic Coordinates $\left(\times 10^{4}\right)$ and Equivalent Isotropic Displacement Parameters $\left(\AA^{2} \times 10^{3}\right)$ for cyclothiocurvularin $A$. $U_{e q}$ is defined as $1 / 3$ of of the trace of the orthogonalised

$\mathrm{U}_{\mathrm{IJ}}$ tensor.

\begin{tabular}{|c|c|c|c|c|}
\hline Atom & $x$ & $y$ & $z$ & $\mathbf{U}(\mathbf{e q})$ \\
\hline $\mathrm{S} 1$ & $7270.7(9)$ & $2190.5(6)$ & $5890.3(4)$ & $24.6(2)$ \\
\hline $\mathrm{O} 1$ & $1269(3)$ & $2348.5(17)$ & $7094.4(11)$ & $28.9(7)$ \\
\hline $\mathrm{O} 2$ & $1770(3)$ & $5496.4(18)$ & $6857.9(13)$ & $34.3(7)$ \\
\hline $\mathrm{O} 3$ & $6064(3)$ & $4827.6(17)$ & $6701.5(13)$ & $34.9(8)$ \\
\hline $\mathrm{O} 4$ & $5776(3)$ & $3023.4(18)$ & $7653.2(11)$ & $32.4(8)$ \\
\hline $\mathrm{O} 5$ & $3085(2)$ & $2430.7(15)$ & $6628.9(10)$ & $22.5(6)$ \\
\hline O6 & $7569(2)$ & $2102.0(16)$ & $7248.1(10)$ & $23.3(6)$ \\
\hline $\mathrm{O} 7$ & $8969(3)$ & $3949.8(18)$ & $7004.5(12)$ & $32.6(7)$ \\
\hline $\mathrm{O} 8$ & $8807(3)$ & $3183.6(19)$ & $7765.5(11)$ & $36.7(8)$ \\
\hline $\mathrm{C} 1$ & $2341(4)$ & $2552(2)$ & $7073.1(16)$ & $23.7(9)$ \\
\hline $\mathrm{C} 2$ & 3035 ( 4) & $2987(3)$ & $7528.3(16)$ & $26.6(10)$ \\
\hline $\mathrm{C} 3$ & $3419(4)$ & $3770(2)$ & $7285.7(15)$ & $21.2(9)$ \\
\hline $\mathrm{C} 4$ & $2483(4)$ & $4290(2)$ & $7189.9(15)$ & $24.9(9)$ \\
\hline $\mathrm{C} 5$ & $2723(4)$ & $5002(2)$ & $6930.3(15)$ & $24.8(9)$ \\
\hline C6 & 3918 ( 4) & $5175(2)$ & $6755.5(15)$ & $24.4(9)$ \\
\hline $\mathrm{C} 7$ & $4874(4)$ & $4645(2)$ & $6843.8(15)$ & $23.7(9)$ \\
\hline $\mathrm{C} 8$ & $4659(4)$ & 3931 (2) & $7106.4(15)$ & $21.2(9)$ \\
\hline C9 & $5672(4)$ & $3373(2)$ & $7204.0(16)$ & $21.3(9)$ \\
\hline C10 & $6568(4)$ & $3170(2)$ & $6718.3(15)$ & $21.4(9)$ \\
\hline C11 & $5970(3)$ & $2622(2)$ & $6286.1(15)$ & $19.8(9)$ \\
\hline
\end{tabular}




\begin{tabular}{|c|c|c|c|c|}
\hline $\mathrm{C} 12$ & $5070(4)$ & $2998(2)$ & $5870.6(16)$ & $22.5(9)$ \\
\hline C13 & $4429(4)$ & $2413(3)$ & $5477.4(16)$ & $25.1(10)$ \\
\hline $\mathrm{C} 14$ & $3656(4)$ & $1783(3)$ & $5769.5(16)$ & $28.3(10)$ \\
\hline $\mathrm{C} 15$ & 2557 (4) & $2054(3)$ & $6124.5(15)$ & $26.6(10)$ \\
\hline $\mathrm{C} 16$ & $1729(4)$ & $2628(3)$ & $5817.7(19)$ & $42.2(13)$ \\
\hline $\mathrm{C} 17$ & $8456(4)$ & $2529(3)$ & $6380.9(16)$ & $26.6(10)$ \\
\hline C18 & $7782(4)$ & $2780(2)$ & $6919.1(15)$ & $21.7(8)$ \\
\hline C19 & $8590(4)$ & 3368 (3) & $7236.2(17)$ & $26.6(9)$ \\
\hline $\mathrm{S} 2$ & $8627.1(13)$ & $93.9(8)$ & $4709.0(4)$ & $41.7(3)$ \\
\hline O9 & $4458(16)$ & $-1718(8)$ & $5153(6)$ & $42(3)$ \\
\hline $\mathrm{O} 10$ & $3019(3)$ & $992.1(17)$ & $7361.9(12)$ & $30.9(7)$ \\
\hline 011 & $7179(3)$ & $636.4(16)$ & $6802.1(11)$ & $26.1(6)$ \\
\hline $\mathrm{O} 12$ & $6621(3)$ & $-1291.6(18)$ & $6014.5(13)$ & $40.7(8)$ \\
\hline O13A & $3850(90)$ & $-490(30)$ & $5210(20)$ & $32(3)$ \\
\hline $\mathrm{O} 13$ & $3810(70)$ & $-420(20)$ & $5249(19)$ & $32(3)$ \\
\hline O14 & $8894(3)$ & $-1250.0(18)$ & $5581.0(13)$ & $38.0(8)$ \\
\hline $\mathrm{O} 15$ & $9604(3)$ & $-972(2)$ & $6648.5(13)$ & $42.8(8)$ \\
\hline 016 & $9825(3)$ & $294.3(19)$ & $6484.6(12)$ & $37.1(8)$ \\
\hline $\mathrm{C} 20$ & $4180(30)$ & $-1154(18)$ & $5454(11)$ & $35(4)$ \\
\hline $\mathrm{C} 21$ & $3999(4)$ & $-1167(3)$ & $6085.3(15)$ & $28.7(10)$ \\
\hline $\mathrm{C} 22$ & 4407 ( 4) & $-452(2)$ & $6411.9(15)$ & $25.6(9)$ \\
\hline $\mathrm{C} 23$ & $3524(4)$ & $-59(2)$ & $6719.4(15)$ & $26.5(9)$ \\
\hline $\mathrm{C} 24$ & $3849(4)$ & $592(2)$ & $7044.8(15)$ & $24.0(9)$ \\
\hline $\mathrm{C} 25$ & $5063(4)$ & $844(2)$ & $7063.1(15)$ & $24.5(9)$ \\
\hline
\end{tabular}




\begin{tabular}{|c|c|c|c|c|}
\hline $\mathrm{C} 26$ & $5960(4)$ & $450(2)$ & $6754.4(15)$ & $22.4(9)$ \\
\hline $\mathrm{C} 27$ & $5658(4)$ & $-190(2)$ & $6411.5(15)$ & $23.0(9)$ \\
\hline $\mathrm{C} 28$ & $6620(4)$ & $-585(3)$ & $6078.0(16)$ & $26.2(9)$ \\
\hline C29 & $7585(4)$ & $-115(2)$ & $5742.7(15)$ & $24.1(9)$ \\
\hline $\mathrm{C} 30$ & $7200(4)$ & $-100(3)$ & $5114.2(15)$ & $29.6(10)$ \\
\hline C31 & $6198(4)$ & $495(3)$ & $4983.4(15)$ & $28.6(10)$ \\
\hline $\mathrm{C} 32$ & $5657(5)$ & $400(3)$ & $4384.0(17)$ & $35.3(11)$ \\
\hline $\mathrm{C} 33$ & $4990(20)$ & $-350(20)$ & $4295(16)$ & $34(3)$ \\
\hline C34 & $3580(30)$ & $-335(18)$ & 4631 (11) & $31(3)$ \\
\hline $\mathrm{C} 35$ & $2870(40)$ & $239(17)$ & $4496(16)$ & $39(4)$ \\
\hline $\mathrm{C} 36$ & $9639(4)$ & $-11(3)$ & $5317.4(17)$ & $37.5(11)$ \\
\hline C37 & $8903(4)$ & $-465(3)$ & $5757.4(17)$ & $31.1(10)$ \\
\hline C38 & $9482(4)$ & $-401(3)$ & $6346.5(18)$ & $32(1)$ \\
\hline O9A & $3910(18)$ & $-1706(12)$ & $5158(8)$ & $42(3)$ \\
\hline $\mathrm{C} 20 \mathrm{~A}$ & $3880(40)$ & $-1120(20)$ & $5432(15)$ & $35(4)$ \\
\hline $\mathrm{C} 33 \mathrm{~A}$ & $4760(30)$ & $-330(30)$ & $4300(20)$ & $34(3)$ \\
\hline C34A & $3760(40)$ & $-450(20)$ & $4576(15)$ & $31(3)$ \\
\hline $\mathrm{C} 35 \mathrm{~A}$ & $2810(50)$ & $390(20)$ & $4550(20)$ & $39(4)$ \\
\hline $\mathrm{O} 20$ & $5309(7)$ & $-2456(4)$ & $4072(3)$ & $41.6(15)$ \\
\hline C40 & $6322(13)$ & $-3019(7)$ & $4184(5)$ & $52(3)$ \\
\hline O17 & $6491(6)$ & $6224(4)$ & $6273(3)$ & $59.1(18)$ \\
\hline O17A & $6060(20)$ & $6149(13)$ & $6020(9)$ & $59.1(18)$ \\
\hline C39A & $6610(40)$ & $6830(12)$ & $6255(12)$ & $76(8)$ \\
\hline $\mathrm{O} 20 \mathrm{~A}$ & $6490(15)$ & $-2689(9)$ & $4747(5)$ & $41.6(15)$ \\
\hline
\end{tabular}




$\begin{array}{lcrrr}\text { C40A } & 6070(40) & -2750(20) & 4145(10) & 52(3) \\ \text { O19 } & 4342(5) & -3188(3) & 5630.2(19) & 67.4(14) \\ \text { O21A } & 4059(10) & -1688(4) & 3190(3) & 54(2) \\ \text { O18 } & 2525(3) & -3119(2) & 6482.7(14) & 49.3(9) \\ \text { O22 } & 4292(3) & -359.0(19) & 2499.1(11) & 32.6(7) \\ \text { O23 } & 3488(9) & -2167(6) & 3934(4) & 41(2) \\ \text { O21 } & 4961(8) & -1733(4) & 2983(3) & 42.8(18)\end{array}$

Table S2. Anisotropic Displacement Parameters $\left(\AA^{2} \times 10^{3}\right)$ for cyclothiocurvularin A. The Anisotropic displacement factor exponent takes the form: $-2 \pi^{2}\left[h^{2} a^{* 2} U_{11}+2 h k a * b * U_{12}+\ldots\right]$.

\begin{tabular}{|c|c|c|c|c|c|c|}
\hline Atom & $\mathrm{U}_{11}$ & $\mathbf{U}_{22}$ & $\mathbf{U}_{33}$ & $\mathbf{U}_{23}$ & $\mathbf{U}_{13}$ & $\mathbf{U}_{12}$ \\
\hline S1 & $24.0(5)$ & $28.0(6)$ & $21.8(5)$ & $-3.8(5)$ & $0.7(4)$ & $2.5(5)$ \\
\hline $\mathrm{O} 1$ & $23.6(15)$ & $26.7(17)$ & $36.3(15)$ & $4.0(13)$ & $5.9(12)$ & $-2.5(14)$ \\
\hline $\mathrm{O} 2$ & $31.9(17)$ & $25.4(17)$ & $45.7(18)$ & $1.7(15)$ & $-2.6(14)$ & $5.2(15)$ \\
\hline $\mathrm{O} 3$ & $34.5(17)$ & $17.0(15)$ & $53.1(19)$ & $1.7(15)$ & $18.7(15)$ & $-0.1(14)$ \\
\hline $\mathrm{O} 4$ & $36.0(17)$ & $39(2)$ & $22.1(14)$ & $3.8(14)$ & $4.3(13)$ & $9.1(16)$ \\
\hline $\mathrm{O} 5$ & $22.9(14)$ & $22.9(15)$ & $21.7(13)$ & $-1.6(12)$ & $2.8(11)$ & $-2.8(13)$ \\
\hline O6 & $24.8(15)$ & $23.3(15)$ & $21.9(13)$ & $0.8(12)$ & $0.0(12)$ & $-1.9(13)$ \\
\hline $\mathrm{O} 7$ & $34.8(17)$ & $24.9(16)$ & $38.1(16)$ & $-1.4(14)$ & $-2.8(13)$ & $-10.8(15)$ \\
\hline O8 & $40(2)$ & $42(2)$ & $27.7(15)$ & $-4.6(14)$ & $-5.6(14)$ & $-18.3(17)$ \\
\hline $\mathrm{C} 1$ & $25(2)$ & $19(2)$ & $27(2)$ & $7.4(17)$ & $4.2(17)$ & $1.8(19)$ \\
\hline $\mathrm{C} 2$ & $29(2)$ & $26(2)$ & $25(2)$ & $-2.1(18)$ & $7.6(17)$ & $0(2)$ \\
\hline $\mathrm{C} 3$ & $25(2)$ & $20(2)$ & $18.5(18)$ & $-5.2(17)$ & $1.7(16)$ & $-0.1(18)$ \\
\hline $\mathrm{C} 4$ & $25(2)$ & $23(2)$ & $27(2)$ & $-3.8(18)$ & $-1.5(18)$ & $-0.1(18)$ \\
\hline $\mathrm{C} 5$ & $29(2)$ & $22(2)$ & $22.9(19)$ & $-5.3(18)$ & $-3.9(17)$ & $3(2)$ \\
\hline
\end{tabular}




\begin{tabular}{|c|c|c|c|c|c|c|}
\hline C6 & $35(2)$ & $16(2)$ & $21.7(19)$ & $-1.9(17)$ & $0.2(17)$ & $-1.7(18)$ \\
\hline $\mathrm{C} 7$ & $31(2)$ & $20(2)$ & $20.3(18)$ & $-6.3(17)$ & $4.7(17)$ & $-3.1(19)$ \\
\hline $\mathrm{C} 8$ & $23(2)$ & $22(2)$ & $19.5(18)$ & $-3.9(17)$ & $1.0(16)$ & $-1.0(18)$ \\
\hline C9 & $24(2)$ & $20(2)$ & $20.3(19)$ & $-4.8(18)$ & $0.0(16)$ & $-4.0(18)$ \\
\hline $\mathrm{C} 10$ & $21(2)$ & $19(2)$ & $23.4(19)$ & $3.2(17)$ & $1.9(16)$ & $-2 \cdot 3(18)$ \\
\hline C11 & $19.5(19)$ & $19(2)$ & $21.1(18)$ & $-0.1(16)$ & $1.5(15)$ & $-2.2(18)$ \\
\hline $\mathrm{C} 12$ & $22(2)$ & $26(2)$ & $19.7(18)$ & $5.0(18)$ & $2.1(16)$ & $-0.7(18)$ \\
\hline C13 & $24(2)$ & $30(2)$ & $20.5(19)$ & $1.9(18)$ & $-1.0(16)$ & $3(2)$ \\
\hline $\mathrm{C} 14$ & $29(2)$ & $33(3)$ & $22.7(19)$ & $-4.6(18)$ & $-1.8(18)$ & $-6(2)$ \\
\hline $\mathrm{C} 15$ & $25(2)$ & $33(3)$ & $22.0(19)$ & $-1.0(18)$ & $-1.8(16)$ & $-8(2)$ \\
\hline $\mathrm{C} 16$ & $26(2)$ & $65(4)$ & $35(2)$ & $14(3)$ & $0.8(19)$ & $6(2)$ \\
\hline $\mathrm{C} 17$ & $22(2)$ & $27(2)$ & $31(2)$ & $-0.3(18)$ & $3.1(17)$ & $-2.7(19)$ \\
\hline C18 & $20.8(19)$ & $19(2)$ & $25.7(19)$ & $1.1(17)$ & $-0.4(15)$ & $-3.5(18)$ \\
\hline C19 & $20(2)$ & $31(2)$ & $29(2)$ & $-4.9(19)$ & $0.0(18)$ & $-1(2)$ \\
\hline S2 & $49.9(8)$ & $48.9(8)$ & $26.3(5)$ & $6.6(6)$ & $9.1(5)$ & $3.4(7)$ \\
\hline O9 & $66(8)$ & $30(2)$ & $30.1(18)$ & $-0.5(16)$ & $2(6)$ & $-15(6)$ \\
\hline $\mathrm{O} 10$ & $30.4(17)$ & $24.3(16)$ & $38.1(16)$ & $0.8(14)$ & $7.3(14)$ & $-2.8(15)$ \\
\hline O11 & $30.5(16)$ & $22.5(16)$ & $25.5(14)$ & $-0.9(13)$ & $-1.4(13)$ & $-2.4(14)$ \\
\hline $\mathrm{O} 12$ & $50(2)$ & $18.8(16)$ & $53.4(19)$ & $2.4(15)$ & $11.4(16)$ & $-1.2(16)$ \\
\hline O13A & $49(4)$ & $27(5)$ & $19(5)$ & $0(4)$ & $-10(4)$ & $-10(5)$ \\
\hline O13 & $49(4)$ & $27(5)$ & $19(5)$ & $0(4)$ & $-10(4)$ & $-10(5)$ \\
\hline O14 & $48(2)$ & $25.3(17)$ & $41.2(17)$ & $-1.6(15)$ & $8.0(15)$ & $6.8(16)$ \\
\hline $\mathrm{O} 15$ & $43(2)$ & $36.4(19)$ & $49.2(19)$ & $15.5(17)$ & $-9.5(16)$ & $5.2(17)$ \\
\hline O16 & $41.6(19)$ & $39(2)$ & $30.8(15)$ & $2.3(15)$ & $-2.5(15)$ & $2.6(17)$ \\
\hline
\end{tabular}




\begin{tabular}{|c|c|c|c|c|c|c|}
\hline $\mathrm{C} 20$ & $50(13)$ & $26(3)$ & $28(3)$ & $2(2)$ & $-9(5)$ & $-10(5)$ \\
\hline $\mathrm{C} 21$ & $41(3)$ & $23(2)$ & $22.4(19)$ & $2.4(17)$ & $-2.8(18)$ & $-7(2)$ \\
\hline $\mathrm{C} 22$ & $37(2)$ & $22(2)$ & $18.1(18)$ & $8.3(17)$ & $-5.4(17)$ & $-3(2)$ \\
\hline $\mathrm{C} 23$ & $30(2)$ & $24(2)$ & $26.0(19)$ & $7.2(18)$ & $-2.8(17)$ & $-1.5(19)$ \\
\hline $\mathrm{C} 24$ & $33(2)$ & $16(2)$ & $23.2(19)$ & $5.5(17)$ & $1.7(18)$ & $2.6(19)$ \\
\hline $\mathrm{C} 25$ & $34(2)$ & $19(2)$ & $20.5(19)$ & $2.6(17)$ & $-1.5(17)$ & $-1.1(19)$ \\
\hline $\mathrm{C} 26$ & $28(2)$ & $19(2)$ & $19.9(18)$ & $7.5(17)$ & $-4.5(17)$ & $1.9(18)$ \\
\hline $\mathrm{C} 27$ & $30(2)$ & $21(2)$ & $18.5(18)$ & $6.1(17)$ & $-2.5(16)$ & $0.6(18)$ \\
\hline $\mathrm{C} 28$ & $36(2)$ & $21(2)$ & $21.5(19)$ & $2.2(18)$ & $-3.9(17)$ & $1(2)$ \\
\hline C29 & $31(2)$ & $20(2)$ & $21.2(18)$ & $1.6(16)$ & $0.0(16)$ & $2.2(19)$ \\
\hline $\mathrm{C} 30$ & $41(2)$ & $24(2)$ & $23.7(19)$ & $2.3(18)$ & $1.6(18)$ & $0(2)$ \\
\hline C31 & $40(3)$ & $23(2)$ & $23(2)$ & $3.1(18)$ & $-2.9(18)$ & $-2(2)$ \\
\hline $\mathrm{C} 32$ & $49(3)$ & $28(2)$ & $28(2)$ & $4(2)$ & $-7(2)$ & $-1(2)$ \\
\hline C33 & $49(7)$ & $31(3)$ & $22(2)$ & $-1(2)$ & $-6(5)$ & $-4(6)$ \\
\hline C34 & $48(7)$ & $28(8)$ & $18(4)$ & $-1(4)$ & $-9(4)$ & $-5(5)$ \\
\hline C35 & $48(5)$ & $37(10)$ & $31(6)$ & $1(7)$ & $-15(4)$ & $-3(6)$ \\
\hline $\mathrm{C} 36$ & $38(3)$ & $39(3)$ & $36(2)$ & $6(2)$ & $8(2)$ & $6(2)$ \\
\hline C37 & $35(2)$ & $27(2)$ & $31(2)$ & $0.3(19)$ & $3.7(18)$ & $6(2)$ \\
\hline C38 & $25(2)$ & $36(3)$ & $35(2)$ & $4(2)$ & $1.0(19)$ & $7(2)$ \\
\hline O9A & $66(8)$ & $30(2)$ & $30.1(18)$ & $-0.5(16)$ & $2(6)$ & $-15(6)$ \\
\hline C20A & $50(13)$ & $26(3)$ & $28(3)$ & $2(2)$ & $-9(5)$ & $-10(5)$ \\
\hline $\mathrm{C} 33 \mathrm{~A}$ & $49(7)$ & $31(3)$ & $22(2)$ & $-1(2)$ & $-6(5)$ & $-4(6)$ \\
\hline C34A & $48(7)$ & $28(8)$ & $18(4)$ & $-1(4)$ & $-9(4)$ & $-5(5)$ \\
\hline $\mathrm{C} 35 \mathrm{~A}$ & $48(5)$ & $37(10)$ & $31(6)$ & $1(7)$ & $-15(4)$ & $-3(6)$ \\
\hline
\end{tabular}




$\begin{array}{lrrrrrr}\text { O20 } & 48(4) & 37(4) & 40(3) & 5(3) & 1(3) & -8(3) \\ \text { C40 } & 60(7) & 26(8) & 68(5) & 2(5) & 12(4) & -1(6) \\ \text { O17 } & 68(5) & 30(3) & 79(5) & 9(4) & 23(4) & -5(3) \\ \text { O17A } & 68(5) & 30(3) & 79(5) & 9(4) & 23(4) & -5(3) \\ \text { C39A } & 130(20) & 23(9) & 75(15) & 12(10) & -20(17) & 3(12) \\ \text { O20A } & 48(4) & 37(4) & 40(3) & 5(3) & 1(3) & -8(3) \\ \text { C40A } & 60(7) & 26(8) & 68(5) & 2(5) & 12(4) & -1(6) \\ \text { O19 } & 104(4) & 28(2) & 70(3) & 4(2) & 37(3) & -1(3) \\ \text { O21A } & 77(6) & 33(4) & 51(5) & -10(4) & -35(5) & 8(5) \\ \text { O18 } & 50(2) & 34(2) & 64(2) & 8.7(18) & 10.1(19) & 10.9(19) \\ \text { O22 } & 36.3(18) & 28.0(17) & 33.5(16) & -2.8(14) & -4.6(14) & 5.9(17) \\ \text { O23 } & 41(6) & 36(6) & 46(6) & 4(5) & -8(5) & 2(6) \\ \text { O21 } & 39(4) & 36(5) & 53(5) & -1(4) & -13(4) & -1(4)\end{array}$

Table S3. Bond Lengths for cyclothiocurvularin A.

\begin{tabular}{llllll} 
Atom Atom & \multicolumn{1}{c}{ Length/Å } & Atom Atom & Length/Å \\
S1 & C11 & $1.844(4)$ & O12 & C28 & $1.231(5)$ \\
S1 & C17 & $1.822(4)$ & O13A C20A & $1.22(8)$ \\
O1 & C1 & $1.211(5)$ & O13A C34A & $1.49(5)$ \\
O2 & C5 & $1.349(5)$ & O13 & C20 & $1.41(6)$ \\
O3 & C7 & $1.365(5)$ & O13 & C34 & $1.49(4)$ \\
O4 & C9 & $1.225(5)$ & O14 & C37 & $1.418(5)$ \\
O5 & C1 & $1.337(5)$ & O15 & C38 & $1.225(5)$ \\
O5 & C15 & $1.472(4)$ & O16 & C38 & $1.298(6)$
\end{tabular}




\begin{tabular}{|c|c|c|c|c|c|}
\hline O6 & C18 & $1.425(5)$ & $\mathrm{C} 20$ & $\mathrm{C} 21$ & $1.50(3)$ \\
\hline $\mathrm{O} 7$ & C19 & $1.215(5)$ & $\mathrm{C} 21$ & $\mathrm{C} 22$ & $1.521(6)$ \\
\hline $\mathrm{O} 8$ & C19 & $1.310(5)$ & $\mathrm{C} 21$ & C20A & 1.55 (3) \\
\hline $\mathrm{C} 1$ & $\mathrm{C} 2$ & $1.510(6)$ & $\mathrm{C} 22$ & $\mathrm{C} 23$ & $1.378(6)$ \\
\hline $\mathrm{C} 2$ & $\mathrm{C} 3$ & $1.527(6)$ & $\mathrm{C} 22$ & $\mathrm{C} 27$ & $1.425(6)$ \\
\hline C3 & $\mathrm{C} 4$ & $1.371(6)$ & $\mathrm{C} 23$ & $\mathrm{C} 24$ & $1.407(6)$ \\
\hline $\mathrm{C} 3$ & $\mathrm{C} 8$ & $1.431(6)$ & $\mathrm{C} 24$ & $\mathrm{C} 25$ & $1.381(6)$ \\
\hline $\mathrm{C} 4$ & $\mathrm{C} 5$ & $1.398(6)$ & $\mathrm{C} 25$ & $\mathrm{C} 26$ & $1.390(6)$ \\
\hline C5 & C6 & $1.387(6)$ & $\mathrm{C} 26$ & $\mathrm{C} 27$ & $1.409(6)$ \\
\hline C6 & $\mathrm{C} 7$ & $1.396(6)$ & $\mathrm{C} 27$ & $\mathrm{C} 28$ & $1.471(6)$ \\
\hline $\mathrm{C} 7$ & $\mathrm{C} 8$ & $1.400(6)$ & $\mathrm{C} 28$ & $\mathrm{C} 29$ & $1.539(6)$ \\
\hline $\mathrm{C} 8$ & C9 & $1.476(6)$ & $\mathrm{C} 29$ & C30 & $1.540(5)$ \\
\hline C9 & $\mathrm{C} 10$ & $1.540(5)$ & $\mathrm{C} 29$ & C37 & $1.547(6)$ \\
\hline C10 & C11 & $1.534(5)$ & C30 & C31 & $1.525(6)$ \\
\hline $\mathrm{C} 10$ & $\mathrm{C} 18$ & $1.548(5)$ & C31 & C32 & $1.538(5)$ \\
\hline C11 & $\mathrm{C} 12$ & $1.525(5)$ & $\mathrm{C} 32$ & C33 & $1.49(3)$ \\
\hline C12 & C13 & $1.538(6)$ & C32 & C33A & $1.60(4)$ \\
\hline C13 & $\mathrm{C} 14$ & $1.535(6)$ & C33 & C34 & $1.72(5)$ \\
\hline C14 & $\mathrm{C} 15$ & $1.526(6)$ & $\mathrm{C} 34$ & C35 & $1.29(5)$ \\
\hline C15 & C16 & $1.519(6)$ & C36 & C37 & $1.525(6)$ \\
\hline C17 & $\mathrm{C} 18$ & $1.527(5)$ & C37 & C38 & $1.528(6)$ \\
\hline C18 & C19 & $1.534(6)$ & O9A & C20A & $1.20(4)$ \\
\hline S2 & C30 & $1.844(4)$ & C33A & C34A & $1.27(6)$ \\
\hline S2 & C36 & $1.813(5)$ & C34A & C35 & $1.79(7)$ \\
\hline
\end{tabular}



O9 C20
$1.24(3) \quad \mathrm{O} 20 \quad \mathrm{C} 40$
$1.488(12)$
$\begin{array}{lllll}\text { O10 } & \text { C24 } & 1.357(5) & \text { O17AC39A } & 1.43(2)\end{array}$
O11 C26 1.359(5) O20AC40A $1.49(2)$

Table S4. Bond Angles for cyclothiocurvularin A.

\begin{tabular}{|c|c|c|c|c|c|c|c|}
\hline \multicolumn{3}{|c|}{ Atom Atom Atom } & Angle ${ }^{\circ}$ & \multicolumn{3}{|c|}{ Atom Atom Atom } & \multirow{2}{*}{$\begin{array}{c}\text { Angle/ }{ }^{\circ} \\
109(3)\end{array}$} \\
\hline $\mathrm{C} 17$ & $\mathrm{~S} 1$ & $\mathrm{C} 11$ & $94.77(17)$ & O13 & $\mathrm{C} 20$ & $\mathrm{C} 21$ & \\
\hline $\mathrm{C} 1$ & $\mathrm{O} 5$ & $\mathrm{C} 15$ & $118.1(3)$ & $\mathrm{C} 20$ & $\mathrm{C} 21$ & $\mathrm{C} 22$ & $116.9(13)$ \\
\hline O1 & $\mathrm{C} 1$ & O5 & $124.2(4)$ & $\mathrm{C} 22$ & $\mathrm{C} 21$ & $\mathrm{C} 20 \mathrm{~A}$ & $119.1(17)$ \\
\hline $\mathrm{O} 1$ & $\mathrm{C} 1$ & $\mathrm{C} 2$ & $126.1(4)$ & $\mathrm{C} 23$ & $\mathrm{C} 22$ & $\mathrm{C} 21$ & $117.8(4)$ \\
\hline O5 & $\mathrm{C} 1$ & $\mathrm{C} 2$ & $109.7(3)$ & $\mathrm{C} 23$ & $\mathrm{C} 22$ & $\mathrm{C} 27$ & $120.0(4)$ \\
\hline $\mathrm{C} 1$ & $\mathrm{C} 2$ & $\mathrm{C} 3$ & $108.1(3)$ & $\mathrm{C} 27$ & $\mathrm{C} 22$ & $\mathrm{C} 21$ & $122.2(4)$ \\
\hline $\mathrm{C} 4$ & $\mathrm{C} 3$ & $\mathrm{C} 2$ & $116.3(4)$ & $\mathrm{C} 22$ & $\mathrm{C} 23$ & $\mathrm{C} 24$ & $120.6(4)$ \\
\hline $\mathrm{C} 4$ & $\mathrm{C} 3$ & $\mathrm{C} 8$ & $120.9(4)$ & O10 & $\mathrm{C} 24$ & $\mathrm{C} 23$ & $122.9(4)$ \\
\hline $\mathrm{C} 8$ & $\mathrm{C} 3$ & $\mathrm{C} 2$ & $122.5(4)$ & O10 & $\mathrm{C} 24$ & $\mathrm{C} 25$ & $116.6(4)$ \\
\hline $\mathrm{C} 3$ & $\mathrm{C} 4$ & $\mathrm{C} 5$ & $120.8(4)$ & $\mathrm{C} 25$ & $\mathrm{C} 24$ & $\mathrm{C} 23$ & $120.4(4)$ \\
\hline $\mathrm{O} 2$ & $\mathrm{C} 5$ & $\mathrm{C} 4$ & $118.1(4)$ & $\mathrm{C} 24$ & $\mathrm{C} 25$ & $\mathrm{C} 26$ & $119.4(4)$ \\
\hline $\mathrm{O} 2$ & C5 & C6 & $122.3(4)$ & O11 & $\mathrm{C} 26$ & $\mathrm{C} 25$ & $121.0(4)$ \\
\hline C6 & C5 & $\mathrm{C} 4$ & $119.5(4)$ & O11 & $\mathrm{C} 26$ & $\mathrm{C} 27$ & $117.2(4)$ \\
\hline $\mathrm{C} 5$ & C6 & $\mathrm{C} 7$ & $120.0(4)$ & $\mathrm{C} 25$ & $\mathrm{C} 26$ & $\mathrm{C} 27$ & $121.6(4)$ \\
\hline $\mathrm{O} 3$ & $\mathrm{C} 7$ & C6 & $120.4(4)$ & $\mathrm{C} 22$ & $\mathrm{C} 27$ & $\mathrm{C} 28$ & $121.5(4)$ \\
\hline $\mathrm{O} 3$ & $\mathrm{C} 7$ & $\mathrm{C} 8$ & $118.0(4)$ & $\mathrm{C} 26$ & $\mathrm{C} 27$ & $\mathrm{C} 22$ & $118.0(4)$ \\
\hline C6 & $\mathrm{C} 7$ & $\mathrm{C} 8$ & $121.5(4)$ & C26 & $\mathrm{C} 27$ & $\mathrm{C} 28$ & $120.5(4)$ \\
\hline $\mathrm{C} 3$ & $\mathrm{C} 8$ & C9 & $121.3(4)$ & $\mathrm{O} 12$ & $\mathrm{C} 28$ & $\mathrm{C} 27$ & $121.8(4)$ \\
\hline
\end{tabular}




\begin{tabular}{|c|c|c|c|c|c|c|c|}
\hline $\mathrm{C} 7$ & $\mathrm{C} 8$ & $\mathrm{C} 3$ & $117.2(4)$ & $\mathrm{O} 12$ & $\mathrm{C} 28$ & $\mathrm{C} 29$ & $117.4(4)$ \\
\hline $\mathrm{C} 7$ & $\mathrm{C} 8$ & C9 & $121.4(3)$ & $\mathrm{C} 27$ & $\mathrm{C} 28$ & $\mathrm{C} 29$ & $120.5(4)$ \\
\hline $\mathrm{O} 4$ & C9 & $\mathrm{C} 8$ & $121.7(3)$ & $\mathrm{C} 28$ & $\mathrm{C} 29$ & C30 & $108.7(3)$ \\
\hline $\mathrm{O} 4$ & C9 & $\mathrm{C} 10$ & $118.3(4)$ & $\mathrm{C} 28$ & $\mathrm{C} 29$ & C37 & $113.9(3)$ \\
\hline $\mathrm{C} 8$ & C9 & $\mathrm{C} 10$ & $119.9(3)$ & $\mathrm{C} 30$ & $\mathrm{C} 29$ & C37 & $106.0(3)$ \\
\hline C9 & $\mathrm{C} 10$ & $\mathrm{C} 18$ & $113.8(3)$ & $\mathrm{C} 29$ & $\mathrm{C} 30$ & $\mathrm{~S} 2$ & $106.1(3)$ \\
\hline C11 & $\mathrm{C} 10$ & C9 & $111.8(3)$ & C31 & C30 & $\mathrm{S} 2$ & $111.4(3)$ \\
\hline $\mathrm{C} 11$ & $\mathrm{C} 10$ & $\mathrm{C} 18$ & $106.9(3)$ & C31 & C30 & $\mathrm{C} 29$ & $113.4(3)$ \\
\hline $\mathrm{C} 10$ & $\mathrm{C} 11$ & $\mathrm{~S} 1$ & $105.5(3)$ & $\mathrm{C} 30$ & C31 & $\mathrm{C} 32$ & $112.5(4)$ \\
\hline $\mathrm{C} 12$ & $\mathrm{C} 11$ & $\mathrm{~S} 1$ & $109.4(3)$ & C31 & C32 & C33A & $115.2(18)$ \\
\hline $\mathrm{C} 12$ & $\mathrm{C} 11$ & C10 & $115.6(3)$ & C33 & C32 & C31 & $113.9(15)$ \\
\hline C11 & $\mathrm{C} 12$ & $\mathrm{C} 13$ & $113.2(3)$ & $\mathrm{C} 32$ & C33 & $\mathrm{C} 34$ & $111(3)$ \\
\hline C14 & $\mathrm{C} 13$ & $\mathrm{C} 12$ & $116.2(3)$ & $\mathrm{O} 13$ & C34 & C33 & $107(3)$ \\
\hline $\mathrm{C} 15$ & $\mathrm{C} 14$ & $\mathrm{C} 13$ & $116.9(4)$ & $\mathrm{C} 35$ & C34 & $\mathrm{O} 13$ & $115(3)$ \\
\hline $\mathrm{O} 5$ & $\mathrm{C} 15$ & C14 & $106.2(3)$ & $\mathrm{C} 35$ & C34 & C33 & $115(3)$ \\
\hline $\mathrm{O} 5$ & $\mathrm{C} 15$ & $\mathrm{C} 16$ & $108.9(4)$ & $\mathrm{C} 37$ & C36 & $\mathrm{S} 2$ & $106.1(3)$ \\
\hline C16 & $\mathrm{C} 15$ & C14 & $113.3(3)$ & O14 & C37 & $\mathrm{C} 29$ & $111.2(4)$ \\
\hline C18 & $\mathrm{C} 17$ & $\mathrm{~S} 1$ & $106.5(3)$ & $\mathrm{O} 14$ & C37 & C36 & $107.2(3)$ \\
\hline O6 & $\mathrm{C} 18$ & C10 & 112.8 (3) & O14 & C37 & C38 & $109.9(4)$ \\
\hline O6 & C18 & C17 & $107.2(3)$ & C36 & C37 & C29 & $105.2(3)$ \\
\hline O6 & $\mathrm{C} 18$ & C19 & $111.8(3)$ & $\mathrm{C} 36$ & C37 & C38 & $111.6(4)$ \\
\hline C17 & $\mathrm{C} 18$ & $\mathrm{C} 10$ & $105.8(3)$ & C38 & C37 & $\mathrm{C} 29$ & $111.6(3)$ \\
\hline C17 & $\mathrm{C} 18$ & C19 & $108.8(3)$ & $\mathrm{O} 15$ & C38 & O16 & $124.7(4)$ \\
\hline C19 & $\mathrm{C} 18$ & $\mathrm{C} 10$ & $110.0(3)$ & O15 & C38 & C37 & $120.9(4)$ \\
\hline
\end{tabular}




$\begin{array}{lllllll}\text { O7 } & \text { C19 } & \text { O8 } & 124.7(4) & \text { O16 } & \text { C38 C37 } & 114.4(4) \\ \text { O7 } & \text { C19 } & \text { C18 } & 121.3(4) & \text { O13A C20AC21 } & 119(4) \\ \text { O8 } & \text { C19 } & \text { C18 } & 113.9(4) & \text { O9A C20A O13A } & 121(4) \\ \text { C36 } & \text { S2 } & \text { C30 } & 94.28(19) & \text { O9A C20AC21 } & 119(3) \\ \text { C20A } 013 A & \text { C34A } & 118(5) & \text { C34A C33AC32 } & 126(4) \\ \text { C20 } & \text { O13 } & \text { C34 } & 118(3) & \text { O13A C34A C35A } & 96(3) \\ \text { O9 } & \text { C20 } & \text { O13 } & 125(3) & \text { C33A C34A O13A } & 117(5) \\ \text { O9 } & \text { C20 } & \text { C21 } & 126(2) & \text { C33A C34A C35A } & 110(4)\end{array}$

Table S5. Hydrogen Bonds for cyclothiocurvularin A.

\begin{tabular}{|c|c|c|c|c|c|c|}
\hline D & $\mathbf{H}$ & $\mathbf{A}$ & $\mathbf{d}(\mathbf{D}-\mathbf{H}) / \AA$ & $\mathbf{d}(\mathbf{H}-\mathbf{A}) / \AA$ & $\mathbf{d}(\mathbf{D}-\mathbf{A}) / \AA$ & D-H-A/ ${ }^{\circ}$ \\
\hline $\mathrm{O} 2$ & $\mathrm{H} 2$ & $\mathrm{O} 18^{1}$ & 0.84 & 1.86 & $2.679(5)$ & 165.6 \\
\hline $\mathrm{O} 3$ & H3 & $\mathrm{O} 17$ & 0.84 & 1.83 & $2.656(7)$ & 167.4 \\
\hline $\mathrm{O} 3$ & $\mathrm{H} 3$ & O17A & 0.84 & 1.98 & $2.79(2)$ & 163.4 \\
\hline O6 & H6 & $\mathrm{O} 4$ & 0.84 & 2.10 & $2.682(4)$ & 126.1 \\
\hline O8 & $\mathrm{H} 8$ & $\mathrm{O} 15^{2}$ & 0.84 & 1.82 & $2.642(4)$ & 166.0 \\
\hline $\mathrm{C} 2$ & $\mathrm{H} 2 \mathrm{~A}$ & $\mathrm{O} 4$ & 0.99 & 2.23 & $2.974(5)$ & 130.6 \\
\hline $\mathrm{C} 10$ & H10A & $\mathrm{O} 3$ & 1.00 & 2.21 & $2.915(5)$ & 126.6 \\
\hline $\mathrm{O} 10$ & $\mathrm{H} 10$ & $\mathrm{O} 22^{3}$ & 0.84 & 1.92 & $2.743(4)$ & 168.4 \\
\hline O11 & H11 & O6 & 0.84 & 1.94 & $2.775(4)$ & 174.6 \\
\hline $\mathrm{O} 14$ & H14 & $\mathrm{O} 12$ & 0.84 & 1.93 & $2.659(4)$ & 145.2 \\
\hline O16 & H16 & $\mathrm{O} 22^{4}$ & 0.84 & 1.75 & $2.579(4)$ & 171.1 \\
\hline $\mathrm{C} 40$ & $\mathrm{H} 40 \mathrm{~A}$ & $\mathrm{O} 18^{5}$ & 0.98 & 2.11 & $2.833(15)$ & 129.7 \\
\hline $\mathrm{O} 17$ & H17D & $\mathrm{O} 23^{6}$ & 0.85 & 2.03 & $2.747(12)$ & 142.3 \\
\hline
\end{tabular}




\begin{tabular}{|c|c|c|c|c|c|}
\hline $\mathrm{O} 17$ & H17E O19 ${ }^{1}$ & 0.85 & 2.29 & $2.952(9)$ & 135.5 \\
\hline \multicolumn{2}{|c|}{ O17A H17C O19 ${ }^{1}$} & 0.84 & 1.57 & $2.37(3)$ & 157.1 \\
\hline \multicolumn{2}{|c|}{ C39A H39B O21 A 6} & 0.98 & 2.01 & $2.96(4)$ & 162.1 \\
\hline \multicolumn{2}{|c|}{ C40A H40F O14 } & 0.98 & 2.09 & $2.99(4)$ & 151.0 \\
\hline O19 & H19AO9 & 0.85 & 2.06 & $2.781(16)$ & 141.9 \\
\hline O19 & H19A O9A & 0.85 & 2.23 & $2.83(2)$ & 127.3 \\
\hline \multicolumn{2}{|c|}{$\mathrm{O} 21 \mathrm{AH} 21 \mathrm{E} \mathrm{O} 1^{8}$} & 0.85 & 2.06 & $2.848(8)$ & 155.0 \\
\hline O18 & H18A O19 & 0.85 & 1.98 & $2.812(6)$ & 166.9 \\
\hline $\mathrm{O} 18$ & $\mathrm{H} 18 \mathrm{~B} \mathrm{O} 20^{7}$ & 0.85 & 2.06 & $2.902(8)$ & 168.1 \\
\hline $\mathrm{O} 22$ & $\mathrm{H} 22 \mathrm{AO} 21 \mathrm{~A}$ & 0.85 & 2.11 & $2.827(9)$ & 142.2 \\
\hline $\mathrm{O} 22$ & $\mathrm{H} 22 \mathrm{AO} 21$ & 0.85 & 1.91 & $2.732(8)$ & 161.4 \\
\hline $\mathrm{O} 22$ & $\mathrm{H} 22 \mathrm{~B} \mathrm{O}^{9}$ & 0.85 & 1.91 & $2.725(4)$ & 160.9 \\
\hline $\mathrm{O} 23$ & H23B O $17^{9}$ & 0.85 & 2.07 & $2.747(12)$ & 135.8 \\
\hline $\mathrm{O} 21$ & $\mathrm{H} 21 \mathrm{GO} 8^{10}$ & 0.85 & 2.27 & $2.884(9)$ & 129.4 \\
\hline $\mathrm{O} 21$ & H21HO20 & 0.85 & 2.16 & $2.881(9)$ & 142.0 \\
\hline $\mathrm{O} 21$ & H21HO23 & 0.85 & 2.07 & $2.850(13)$ & 151.8 \\
\hline
\end{tabular}

${ }^{1}+\mathrm{X}, 1+\mathrm{Y},+\mathrm{Z} ;{ }^{2} 2-\mathrm{X}, 1 / 2+\mathrm{Y}, 3 / 2-\mathrm{Z} ;{ }^{3} 1 / 2-\mathrm{X},-\mathrm{Y}, 1 / 2+\mathrm{Z} ;{ }^{4} 3 / 2-\mathrm{X},-\mathrm{Y}, 1 / 2+\mathrm{Z} ;{ }^{5} 1 / 2+\mathrm{X},-1 / 2-\mathrm{Y}, 1-\mathrm{Z} ;{ }^{6} 1 / 2+\mathrm{X}, 1 / 2-\mathrm{Y}, 1-\mathrm{Z} ;{ }^{7}-1 / 2+\mathrm{X},-1 / 2-\mathrm{Y}, 1-\mathrm{Z} ;{ }^{8} 1 / 2-\mathrm{X},-\mathrm{Y},-1 / 2+\mathrm{Z} ;{ }^{9}-$ $1 / 2+X, 1 / 2-Y, 1-Z ;{ }^{10} 3 / 2-X,-Y,-1 / 2+Z$

Table S6. Torsion Angles for cyclothiocurvularin A.
A $\quad$ B $\quad$ C $\quad$ D
Angle $/^{\circ}$ A $\quad$ B $\quad$ C
D
Angle $/^{\circ}$

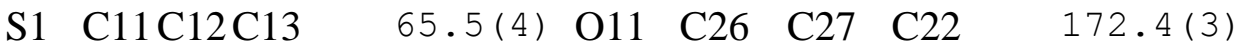
$\begin{array}{llllllll}\text { S1 } & \mathrm{C} 17 \mathrm{C} 18 \mathrm{O} 6 & 82.7(3) & \mathrm{O} 11 & \mathrm{C} 26 & \mathrm{C} 27 & \mathrm{C} 28 & -5.9(5)\end{array}$
$\begin{array}{llllllll}\mathrm{S} 1 & \mathrm{C} 17 \mathrm{C} 18 \mathrm{C} 10 & -38.0(4) & \mathrm{O} 12 & \mathrm{C} 28 & \mathrm{C} 29 & \mathrm{C} 30 & 72.8(5)\end{array}$ 


\begin{tabular}{|c|c|c|c|c|c|c|c|c|c|}
\hline $\mathrm{S} 1$ & $\mathrm{C} 17$ & $7 \mathrm{C} 18$ & $8 \mathrm{C} 19$ & $-156.2(3)$ & $\mathrm{O} 12$ & $\mathrm{C} 28$ & $\mathrm{C} 29$ & $\mathrm{C} 37$ & $-45.1(5)$ \\
\hline $\mathrm{O} 1$ & $\mathrm{C} 1$ & $\mathrm{C} 2$ & $\mathrm{C} 3$ & $-117.1(4)$ & $\mathrm{O} 13$ & $\mathrm{C} 20$ & $\mathrm{C} 21$ & $\mathrm{C} 22$ & $-43(4)$ \\
\hline 2 & $\mathrm{C} 5$ & C6 & $\mathrm{C} 7$ & $179.6(3)$ & $\mathrm{O} 14$ & C37 & C38 & $\mathrm{O} 15$ & $-16.3(6)$ \\
\hline 3 & $\mathrm{C} 7$ & $\mathrm{C} 8$ & $\mathrm{C} 3$ & $176.1(3)$ & $\mathrm{O} 14$ & C37 & C38 & $\mathrm{O} 16$ & $163.1(4)$ \\
\hline 3 & $\mathrm{C} 7$ & $\mathrm{C} 8$ & C9 & $-2.9(5)$ & $\mathrm{C} 20$ & $\mathrm{O} 13$ & C34 & $\mathrm{C} 33$ & $-74(6)$ \\
\hline 4 & $\mathrm{C} 9$ & $\mathrm{C} 1 \mathrm{C}$ & C11 & $99.4(4)$ & $\mathrm{C} 20$ & $\mathrm{O} 13$ & C34 & $\mathrm{C} 35$ & $158(5)$ \\
\hline 4 & C9 & $\mathrm{C} 1 \mathrm{C}$ & C18 & $-21.8(5)$ & $\mathrm{C} 20$ & $\mathrm{C} 21$ & $\mathrm{C} 22$ & $\mathrm{C} 23$ & $119.9(12)$ \\
\hline 5 & $\mathrm{C} 1$ & $\mathrm{C} 2$ & C3 & $61.8(4)$ & $\mathrm{C} 20$ & $\mathrm{C} 21$ & $\mathrm{C} 22$ & $\mathrm{C} 27$ & $-60.7(12)$ \\
\hline & C18 & $\mathrm{C} 1 \mathrm{C}$ & 907 & $172.9(4)$ & $\mathrm{C} 21$ & $\mathrm{C} 22$ & $\mathrm{C} 23$ & $\mathrm{C} 24$ & $178.0(3)$ \\
\hline 6 & C18 & $3 \mathrm{C} 1 \mathrm{~S}$ & 908 & $-7.7(5)$ & $\mathrm{C} 21$ & $\mathrm{C} 22$ & $\mathrm{C} 27$ & $\mathrm{C} 26$ & $-176.2(3)$ \\
\hline 1 & $\mathrm{O} 5$ & $\mathrm{C} 15$ & $5 \mathrm{C} 14$ & $-163.9(3)$ & $\mathrm{C} 21$ & $\mathrm{C} 22$ & $\mathrm{C} 27$ & $\mathrm{C} 28$ & $2.0(6)$ \\
\hline 1 & $\mathrm{O} 5$ & $\mathrm{C} 15$ & $5 \mathrm{C} 16$ & $73.7(4)$ & $\mathrm{C} 22$ & $\mathrm{C} 21$ & \multicolumn{2}{|c|}{ C20A 013A } & $-15(7)$ \\
\hline 1 & $\mathrm{C} 2$ & $\mathrm{C} 3$ & $\mathrm{C} 4$ & $69.9(4)$ & $\mathrm{C} 22$ & $\mathrm{C} 21$ & \multicolumn{2}{|c|}{ C20A O9A } & $159(3)$ \\
\hline $\mathrm{C} 1$ & $\mathrm{C} 2$ & $\mathrm{C} 3$ & $\mathrm{C} 8$ & $-103.3(4)$ & $\mathrm{C} 22$ & $\mathrm{C} 23$ & $\mathrm{C} 24$ & $\mathrm{O} 10$ & $-179.0(3)$ \\
\hline $\mathrm{C} 2$ & $\mathrm{C} 3$ & $\mathrm{C} 4$ & $\mathrm{C} 5$ & $-175.3(3)$ & $\mathrm{C} 22$ & $\mathrm{C} 23$ & $\mathrm{C} 24$ & $\mathrm{C} 25$ & $-0.4(6)$ \\
\hline 2 & $\mathrm{C} 3$ & $\mathrm{C} 8$ & $\mathrm{C} 7$ & $174.3(3)$ & $\mathrm{C} 22$ & $\mathrm{C} 27$ & $\mathrm{C} 28$ & $\mathrm{O} 12$ & $-35.6(6)$ \\
\hline $\mathrm{C} 2$ & $\mathrm{C} 3$ & $\mathrm{C} 8$ & C9 & $-6.8(6)$ & $\mathrm{C} 22$ & $\mathrm{C} 27$ & $\mathrm{C} 28$ & $\mathrm{C} 29$ & $137.8(4)$ \\
\hline $\mathrm{C} 3$ & $\mathrm{C} 4$ & $\mathrm{C} 5$ & $\mathrm{O} 2$ & $-178.6(3)$ & $\mathrm{C} 23$ & $\mathrm{C} 22$ & $\mathrm{C} 27$ & $\mathrm{C} 26$ & $3.2(5)$ \\
\hline C3 & $\mathrm{C} 4$ & $\mathrm{C} 5$ & C6 & $1.6(6)$ & $\mathrm{C} 23$ & $\mathrm{C} 22$ & $\mathrm{C} 27$ & $\mathrm{C} 28$ & $-178.6(3)$ \\
\hline C3 & $\mathrm{C} 8$ & C9 & $\mathrm{O} 4$ & $-42.1(6)$ & $\mathrm{C} 23$ & $\mathrm{C} 24$ & $\mathrm{C} 25$ & $\mathrm{C} 26$ & $0.3(6)$ \\
\hline $\mathrm{C}$ & $\mathrm{C} 8$ & C9 & $\mathrm{C} 10$ & $132.9(4)$ & $\mathrm{C} 24$ & $\mathrm{C} 25$ & $\mathrm{C} 26$ & $\mathrm{O} 11$ & $-173.9(3)$ \\
\hline $\mathrm{C} 4$ & $\mathrm{C} 3$ & $\mathrm{C} 8$ & $\mathrm{C} 7$ & $1.5(5)$ & $\mathrm{C} 24$ & $\mathrm{C} 25$ & C26 & $\mathrm{C} 27$ & $1.6(6)$ \\
\hline $\mathrm{C} 4$ & $\mathrm{C} 3$ & $\mathrm{C} 8$ & $\mathrm{C} 9$ & $-179.6(3)$ & $\mathrm{C} 25$ & $\mathrm{C} 26$ & $\mathrm{C} 27$ & $\mathrm{C} 22$ & $-3.3(5)$ \\
\hline & 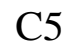 & C6 & $\mathrm{C}$ & $-0.6(6)$ & C25 & 26 & $\mathrm{C} 27$ & $\mathrm{C} 2$ & $17 \varepsilon$ \\
\hline
\end{tabular}




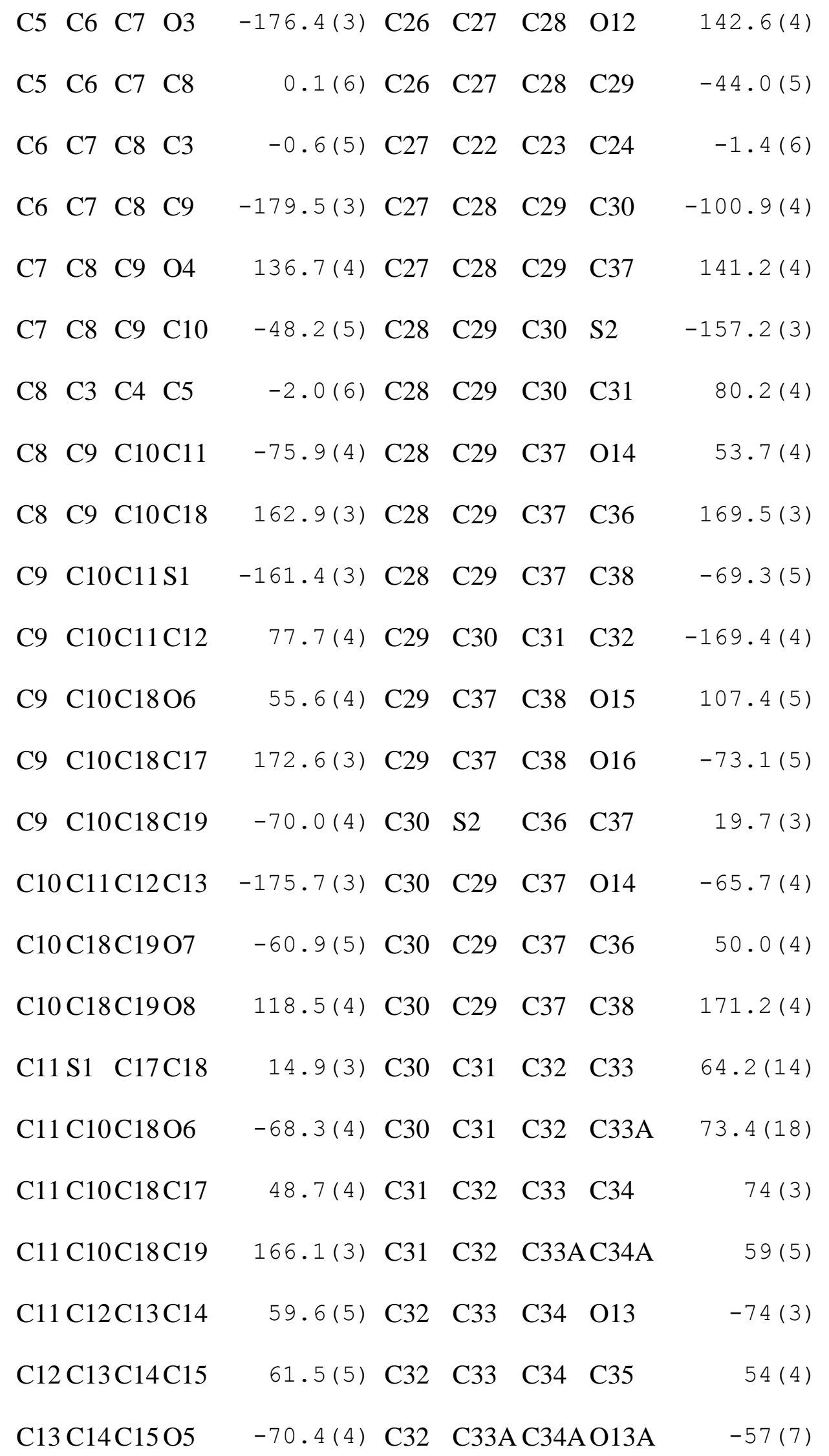




\begin{tabular}{|c|c|c|c|c|c|c|}
\hline C13 C14 C15 C16 & \multirow{2}{*}{$\begin{array}{c}49.1(5) \\
2.4(6)\end{array}$} & \multirow{2}{*}{$\begin{array}{l}\text { C32 } \\
\text { C34 }\end{array}$} & \multicolumn{3}{|c|}{ C33A C34A C35A } & \multirow{2}{*}{$\begin{array}{l}51(7) \\
9(8)\end{array}$} \\
\hline $\mathrm{C} 15 \mathrm{O} 5 \mathrm{C} 1 \mathrm{O} 1$ & & & $\mathrm{O} 13$ & $\mathrm{C} 20$ & O9 & \\
\hline $\mathrm{C} 15 \mathrm{O} 5 \mathrm{C} 1 \mathrm{C} 2$ & $-176.4(3)$ & C34 & $\mathrm{O} 13$ & $\mathrm{C} 20$ & $\mathrm{C} 21$ & $-163(4)$ \\
\hline C17 S1 C11 C10 & $12.4(3)$ & C36 & $\mathrm{S} 2$ & $\mathrm{C} 30$ & $\mathrm{C} 29$ & $8.7(3)$ \\
\hline C17 S1 C11 C12 & $137.3(3)$ & C36 & $\mathrm{S} 2$ & C30 & C31 & $132.6(3)$ \\
\hline C17 C18C19O7 & $54.7(5)$ & C36 & C37 & $\mathrm{C} 38$ & $\mathrm{O} 15$ & $-135.2(4)$ \\
\hline C17 C18C19O8 & $-125.9(4)$ & C36 & C37 & C38 & O16 & $44.3(5)$ \\
\hline C18 C10C11 S1 & $-36.3(3)$ & C37 & C29 & C30 & $\mathrm{S} 2$ & $-34.4(4)$ \\
\hline C18 C10C11 C12 & $-157.2(3)$ & C37 & C29 & C30 & C31 & $-157.0(4)$ \\
\hline S2 C30C31 C32 & $71.0(4)$ & $\mathrm{C} 20$ & $\mathrm{O} 13$ & $\mathrm{C} 34 \mathrm{~A}$ & C33A & $-100(9)$ \\
\hline S2 C36C37 O14 & $75.9(4)$ & $\mathrm{C} 20$ & $\mathrm{O} 13$ & $\mathrm{C} 34 \mathrm{~A}$ & C35A & $144(8)$ \\
\hline S2 C36C37 C29 & $-42.5(4)$ & $\mathrm{C} 20$ & $\mathrm{C} 21$ & $\mathrm{C} 22$ & $\mathrm{C} 23$ & $105.9(17)$ \\
\hline S2 C36C37 C38 & $-163.7(3)$ & $\mathrm{C} 20$ & $\mathrm{C} 21$ & $\mathrm{C} 22$ & $\mathrm{C} 27$ & $-74.7(18)$ \\
\hline O9 C20C21 C22 & $145(2)$ & C34 & $\mathrm{O} 13$ & $\mathrm{C} 20 \mathrm{~A}$ & $\mathrm{C} 21$ & $179(4)$ \\
\hline $\mathrm{O} 10 \mathrm{C} 24 \mathrm{C} 25 \mathrm{C} 26$ & $179.0(3)$ & C32 & $\mathrm{OO} 13$ & $\mathrm{C} 20$ & O9A & $5(11$ \\
\hline
\end{tabular}

Table S7. Hydrogen Atom Coordinates $\left(\AA \times 10^{4}\right)$ and Isotropic Displacement Parameters $\left(\AA^{2} \times 10^{3}\right)$ for cyclothiocurvularin $A$.

\begin{tabular}{|c|c|c|c|c|}
\hline Atom & $x$ & $y$ & $z$ & $\mathrm{U}(\mathbf{e q})$ \\
\hline $\mathrm{H} 2$ & 2019 & 5892 & 6685 & 52 \\
\hline H3 & 6082 & 5273 & 6559 & 52 \\
\hline H6 & 7226 & 2224 & 7555 & 35 \\
\hline H8 & 9350 & 3480 & 7899 & 55 \\
\hline $\mathrm{H} 2 \mathrm{~A}$ & 3777 & 2691 & 7646 & 2 \\
\hline
\end{tabular}




\begin{tabular}{|c|c|c|c|c|}
\hline $\mathrm{H} 2 \mathrm{~B}$ & 2498 & 3062 & 7864 & 32 \\
\hline $\mathrm{H} 4$ & 1662 & 4165 & 7301 & 30 \\
\hline H6A & 4087 & 5656 & 6575 & 29 \\
\hline H10A & 6790 & 3661 & 6518 & 26 \\
\hline H11A & 5526 & 2202 & 6495 & 24 \\
\hline $\mathrm{H} 12 \mathrm{~A}$ & 5527 & 3379 & 5636 & 27 \\
\hline H12B & 4431 & 3283 & 6087 & 27 \\
\hline H13A & 3882 & 2702 & 5216 & 30 \\
\hline H13B & 5073 & 2156 & 5245 & 30 \\
\hline H14A & 3341 & 1427 & 5474 & 34 \\
\hline H14B & 4213 & 1479 & 6018 & 34 \\
\hline H15 & 2055 & 1595 & 6244 & 32 \\
\hline H16A & 1044 & 2779 & 6066 & 63 \\
\hline H16B & 1398 & 2388 & 5473 & 63 \\
\hline $\mathrm{H} 16 \mathrm{C}$ & 2213 & 3087 & 5715 & 63 \\
\hline H17A & 9051 & 2109 & 6466 & 32 \\
\hline H17B & 8916 & 2970 & 6215 & 32 \\
\hline H10 & 2345 & 750 & 7372 & 46 \\
\hline H11 & 7247 & 1084 & 6938 & 39 \\
\hline H14 & 8233 & -1461 & 5688 & 57 \\
\hline H16 & 10038 & 302 & 6827 & 56 \\
\hline $\mathrm{H} 21 \mathrm{~A}$ & 3185 & -1328 & 6238 & 34 \\
\hline H21B & 4593 & -1586 & 6173 & 34 \\
\hline $\mathrm{H} 21 \mathrm{C}$ & 4455 & -1618 & 6239 & \\
\hline
\end{tabular}




\begin{tabular}{|c|c|c|c|c|}
\hline $\mathrm{H} 21 \mathrm{D}$ & 3109 & -1253 & 6163 & 34 \\
\hline $\mathrm{H} 23$ & 2687 & -229 & 6712 & 32 \\
\hline $\mathrm{H} 25$ & 5282 & 1282 & 7285 & 29 \\
\hline $\mathrm{H} 29$ & 7612 & 426 & 5892 & 29 \\
\hline H30 & 6885 & -624 & 5008 & 35 \\
\hline H31A & 6552 & 1021 & 5021 & 34 \\
\hline H31B & 5522 & 445 & 5265 & 34 \\
\hline H32A & 6352 & 354 & 4112 & 42 \\
\hline H32B & 5192 & 876 & 4287 & 42 \\
\hline $\mathrm{H} 32 \mathrm{C}$ & 5077 & 832 & 4310 & 42 \\
\hline H32D & 6339 & 438 & 4105 & 42 \\
\hline H33A & 5498 & -776 & 4446 & 41 \\
\hline H33B & 4869 & -432 & 3884 & 41 \\
\hline H34 & 3132 & -815 & 4508 & 37 \\
\hline H35A & 2033 & 151 & 4648 & 58 \\
\hline H35B & 2824 & 285 & 4083 & 58 \\
\hline H35C & 3205 & 718 & 4656 & 58 \\
\hline H36A & 10402 & -294 & 5211 & 45 \\
\hline H36B & 9875 & 503 & 5469 & 45 \\
\hline H33C & 4522 & -329 & 3896 & 41 \\
\hline H33D & 5280 & -792 & 4360 & 41 \\
\hline $\mathrm{H} 34 \mathrm{~A}$ & 3314 & -913 & 4423 & 37 \\
\hline H35D & 2112 & 332 & 4818 & 58 \\
\hline H35E & 2485 & 465 & 4169 & \\
\hline
\end{tabular}




\begin{tabular}{|c|c|c|c|c|}
\hline $\mathrm{H} 35 \mathrm{~F}$ & 3296 & 847 & 4662 & 58 \\
\hline $\mathrm{H} 20$ & 5108 & -2237 & 4376 & 62 \\
\hline $\mathrm{H} 40 \mathrm{~A}$ & 7121 & -2783 & 4087 & 77 \\
\hline $\mathrm{H} 40 \mathrm{~B}$ & 6196 & -3483 & 3951 & 77 \\
\hline $\mathrm{H} 40 \mathrm{C}$ & 6318 & -3163 & 4585 & 77 \\
\hline H17D & 7147 & 6461 & 6370 & 89 \\
\hline H17E & 5890 & 6541 & 6261 & 89 \\
\hline $\mathrm{H} 17 \mathrm{C}$ & 5407 & 6271 & 5847 & 89 \\
\hline H39A & 6854 & 7180 & 5948 & 114 \\
\hline H39B & 7348 & 6685 & 6474 & 114 \\
\hline H39C & 6016 & 7090 & 6503 & 114 \\
\hline $\mathrm{H} 20 \mathrm{~A}$ & 6859 & -2266 & 4795 & 62 \\
\hline H40D & 6630 & -3087 & 3934 & 77 \\
\hline $\mathrm{H} 40 \mathrm{E}$ & 6067 & -2232 & 3972 & 77 \\
\hline $\mathrm{H} 40 \mathrm{~F}$ & 5227 & -2962 & 4134 & 77 \\
\hline H19A & 4711 & -2829 & 5450 & 101 \\
\hline H19B & 3625 & -3257 & 5491 & 101 \\
\hline $\mathrm{H} 21 \mathrm{E}$ & 4054 & -2005 & 2916 & 81 \\
\hline $\mathrm{H} 21 \mathrm{~F}$ & 4065 & -1936 & 3502 & 81 \\
\hline H18A & 3002 & -3196 & 6202 & 74 \\
\hline H18B & 1848 & -2922 & 6367 & 74 \\
\hline $\mathrm{H} 22 \mathrm{~A}$ & 4586 & -722 & 2702 & 49 \\
\hline $\mathrm{H} 22 \mathrm{~B}$ & 4366 & 69 & 2674 & 49 \\
\hline $\mathrm{H} 23 \mathrm{~A}$ & 3830 & -1959 & 4221 & \\
\hline
\end{tabular}




\begin{tabular}{|c|c|c|c|c|}
\hline $\mathrm{H} 23 \mathrm{~B}$ & 2709 & -2099 & 3951 & 62 \\
\hline $\mathrm{H} 21 \mathrm{G}$ & 5669 & -1930 & 2921 & 64 \\
\hline $\mathrm{H} 21 \mathrm{H}$ & 4753 & -1818 & 3326 & 54 \\
\hline
\end{tabular}

Table S8. Atomic Occupancy for cyclothiocurvularin A.

\begin{tabular}{|c|c|c|c|c|c|}
\hline Atom & Occupancy & Atom & Occupancy & Atom & Occupancy \\
\hline O9 & $0.57(2)$ & O13A & $0.43(2)$ & O13 & $0.57(2)$ \\
\hline $\mathrm{C} 20$ & $0.57(2)$ & $\mathrm{H} 21 \mathrm{~A}$ & $0.43(2)$ & $\mathrm{H} 21 \mathrm{~B}$ & $0.43(2)$ \\
\hline $\mathrm{H} 21 \mathrm{C}$ & $0.57(2)$ & $\mathrm{H} 21 \mathrm{D}$ & $0.57(2)$ & $\mathrm{H} 32 \mathrm{~A}$ & $0.43(2)$ \\
\hline H32B & $0.43(2)$ & $\mathrm{H} 32 \mathrm{C}$ & $0.57(2)$ & H32D & $0.57(2)$ \\
\hline C33 & $0.57(2)$ & H33A & $0.57(2)$ & H33B & $0.57(2)$ \\
\hline C34 & $0.57(2)$ & H34 & $0.57(2)$ & C35 & $0.57(2)$ \\
\hline $\mathrm{H} 35 \mathrm{~A}$ & $0.57(2)$ & H35B & $0.57(2)$ & $\mathrm{H} 35 \mathrm{C}$ & $0.57(2)$ \\
\hline O9A & $0.43(2)$ & C20A & $0.43(2)$ & C33A & $0.43(2)$ \\
\hline H33C & $0.43(2)$ & H33D & $0.43(2)$ & C34A & $0.43(2)$ \\
\hline H34A & $0.43(2)$ & C35A & $0.43(2)$ & H35D & $0.43(2)$ \\
\hline H35E & $0.43(2)$ & $\mathrm{H} 35 \mathrm{~F}$ & $0.43(2)$ & $\mathrm{O} 20$ & $0.529(5)$ \\
\hline $\mathrm{H} 20$ & $0.529(5)$ & $\mathrm{C} 40$ & $0.529(5)$ & $\mathrm{H} 40 \mathrm{~A}$ & $0.529(5)$ \\
\hline $\mathrm{H} 40 \mathrm{~B}$ & $0.529(5)$ & $\mathrm{H} 40 \mathrm{C}$ & $0.529(5)$ & O17 & 0.75 \\
\hline H17D & 0.75 & H17E & 0.75 & O17A & 0.25 \\
\hline H17C & 0.25 & C39A & 0.25 & H39A & 0.25 \\
\hline H39B & 0.25 & $\mathrm{H} 39 \mathrm{C}$ & 0.25 & O20A & $0.221(5)$ \\
\hline $\mathrm{H} 20 \mathrm{~A}$ & $0.221(5)$ & C40A & $0.221(5)$ & H40D & $0.221(5)$ \\
\hline $\mathrm{H} 40 \mathrm{E}$ & $0.221(5)$ & $\mathrm{H} 40 \mathrm{~F}$ & $0.221(5)$ & O19 & 0.9 \\
\hline
\end{tabular}




$\begin{array}{lrcr}\text { H19A } & 0.9 \mathrm{H} 19 \mathrm{~B} & 0.9 \mathrm{O} 21 \mathrm{~A} & 0.5 \\ \mathrm{H} 21 \mathrm{E} & 0.5 \mathrm{H} 21 \mathrm{~F} & 0.5 \mathrm{O} 23 & 0.3333 \\ \mathrm{H} 23 \mathrm{~A} & 0.3333 \mathrm{H} 23 \mathrm{~B} & 0.3333 \mathrm{O} 21 & 0.5 \\ \mathrm{H} 21 \mathrm{G} & 0.5 \mathrm{H} 21 \mathrm{H} & 0.5 & \end{array}$




\section{Data Collection for dimethoxymethyl ester of cyclothiocurvularin B (9) X-ray analysis}

A colourless plate crystal of $\mathrm{C}_{22} \mathrm{H}_{28} \mathrm{O}_{8} \mathrm{~S}$ having approximate dimensions of $0.03 \times 0.12 \times 0.14 \mathrm{~mm}$ was mounted on a glass fiber. All measurements were made on a Bruker APEX DUO diffractometer with a TRIUMPH curved-crystal monochromator with Mo-K $\alpha$ radiation. The data were collected at a temperature of $-183.0 \pm 0.1^{\circ} \mathrm{C}$ to a maximum $2 \theta$ value of $48.6^{\circ}$. Data were collected in a series of $\phi$ and $\omega$ scans in $0.5^{\circ}$ oscillations using 10.0-second exposures. The crystal-to-detector distance was $59.53 \mathrm{~mm}$.

\section{Data Reduction}

Of the 26253 reflections that were collected, 6912 were unique ( $R_{\text {int }}=0.098$ ); equivalent reflections were merged. Data were collected and integrated using the Bruker SAINT ${ }^{1}$ software package. The linear absorption coefficient, $\mu$, for Mo-K $\alpha$ radiation is $1.97 \mathrm{~cm}^{-1}$. Data were corrected for absorption effects using the multi-scan technique $\left(\mathrm{SADABS}^{2}\right)$, with minimum and maximum transmission coefficients of 0.747 and 0.994 , respectively. The data were corrected for Lorentz and polarization effects.

\section{$\underline{\text { Structure Solution and Refinement }}$}

The structure of dimethoxymethyl ester of cyclothiocurvularin B (9) was solved by direct methods. ${ }^{3}$ The material crystallizes with two crystallographically independent molecules in the asymmetric unit. The

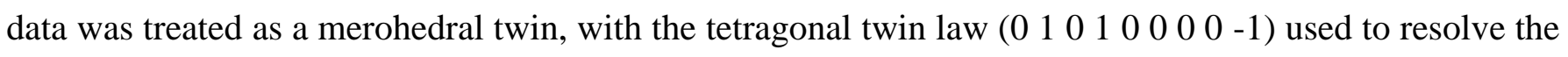
twinning. All non-hydrogen atoms were refined anisotropically. All hydrogen atoms were placed in calculated positions. The refined Flack ${ }^{12}$ parameter is $0.04(7)$. The final cycle of full-matrix leastsquares refinement ${ }^{4}$ on $\mathrm{F}^{2}$ was based on 6912 reflections and 570 variable parameters and converged (largest parameter shift was 0.00 times its esd) with unweighted and weighted agreement factors of:

$$
\begin{gathered}
\mathrm{R} 1(\mathrm{I}>2.00 \sigma(\mathrm{I}))=\Sigma\|\mathrm{Fo}|-| \mathrm{Fc}\| / \Sigma|\mathrm{Fo}|=0.112 \\
\mathrm{wR} 2(\text { all data })=\left[\Sigma\left(\mathrm{w}\left(\mathrm{Fo}^{2}-\mathrm{Fc}^{2}\right)^{2}\right) / \Sigma \mathrm{w}\left(\mathrm{Fo}^{2}\right)^{2}\right]^{1 / 2}=0.304
\end{gathered}
$$

The standard deviation of an observation of unit weight ${ }^{5}$ was 1.07. The weighting scheme was based on counting statistics. The maximum and minimum peaks on the final difference Fourier map corresponded to 0.57 and $-0.604 \mathrm{e}^{-} / \AA^{3}$ respectively.

Neutral atom scattering factors were taken from Cromer and Waber. ${ }^{6}$ Anomalous dispersion effects were included in Fcalc; $;^{7}$ the values for $\Delta \mathrm{f}^{\prime}$ and $\Delta \mathrm{f}^{\prime \prime}$ were those of Creagh and McAuley. ${ }^{8}$ The values for the mass 
attenuation coefficients are those of Creagh and Hubbell. ${ }^{9}$ All refinements were performed using the XL ${ }^{10}$ via the OLEX $2^{11}$ interface.

\section{References}

(1) SAINT. Version 8.34A Bruker AXS Inc., Madison, Wisconsin, USA. (1997-2013).

(2) SADABS 2014/5 - Krause, L., Herbst-Irmer, R., Sheldrick, G. M. \& Stalke, D. (2015). J. Appl. Crystallogr. 48.

(3) SHELXT: Sheldrick, G. M. Acta Cryst. 2015, A71, 3-8.

(4) Least Squares function minimized:

$$
\Sigma w\left(\mathrm{~F}_{\mathrm{o}}^{2}-\mathrm{F}_{\mathrm{c}}\right)^{2}
$$

(5) Standard deviation of an observation of unit weight:

$$
\begin{aligned}
& {\left[\sum w\left(\mathrm{~F}_{\mathrm{O}}{ }^{2}-\mathrm{F}_{\mathrm{C}}{ }^{2}\right)^{2} /\left(\mathrm{N}_{\mathrm{O}}-\mathrm{N}_{\mathrm{V}}\right)\right]^{1 / 2}} \\
& \text { where: } \quad \mathrm{N}_{\mathrm{O}}=\text { number of observations; } \mathrm{N}_{\mathrm{V}}=\text { number of variables }
\end{aligned}
$$

(6) Cromer, D. T.; Waber, J. T. International Tables for X-ray Crystallography, Vol. IV, The Kynoch Press, Birmingham, England, Table 2.2 A (1974).

(7) Ibers, J. A.; Hamilton, W. C. Acta Crystallogr. 1964, 17, 781.

(8) Creagh, D. C.; McAuley, W.J . International Tables for Crystallography, Vol C, (A.J.C. Wilson, ed.), Kluwer Academic Publishers, Boston, Table 4.2.6.8, pages 219-222 (1992).

(9) Creagh, D. C.; Hubbell, J.H. International Tables for Crystallography, Vol C, (A.J.C. Wilson, ed.), Kluwer Academic Publishers, Boston, Table 4.2.4.3, pages 200-206 (1992).

(10) XL Sheldrick, G. M., Acta Cryst. 2008, A64, 112-122.

(11) OLEX2 - V1.2.6 Dolomanov, O.V.; Bourhis, L.J.; Gildea, R.J.; Howard, J.A.K.; Puschmann, H. J. Appl. Cryst. 2009, 42, 339-341.

(12) Parsons, S.; Flack, H. D.; Wagner, T. Acta Cryst. 2013, B69, 249-259. 


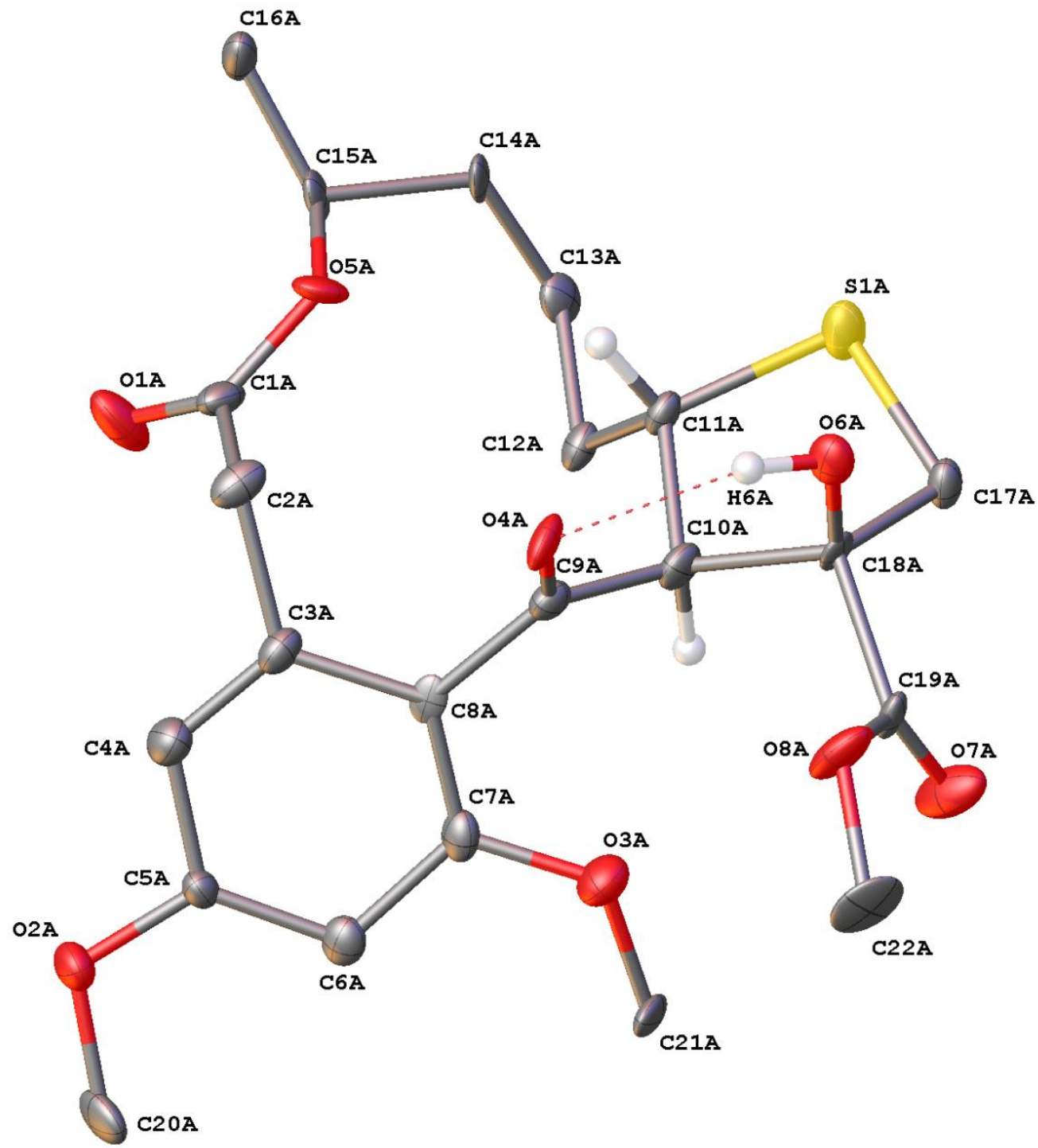

Figure S3. ORTEP-style picture of the molecule A of dimethoxymethyl ester of cyclothiocurvularin B (9). 


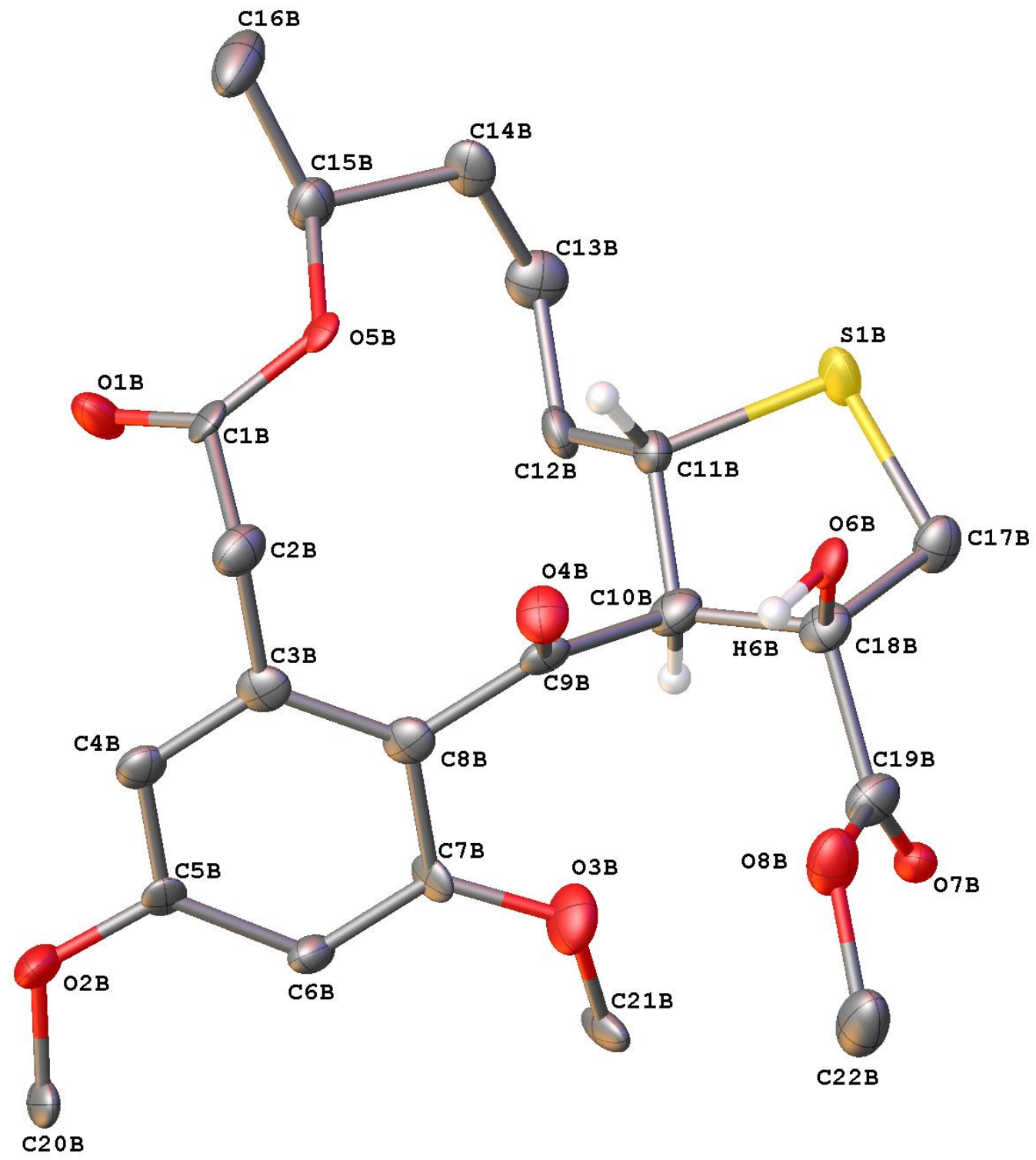

Figure S4. ORTEP-style picture of the molecule B of dimethoxymethyl ester of cyclothiocurvularin B (9). 


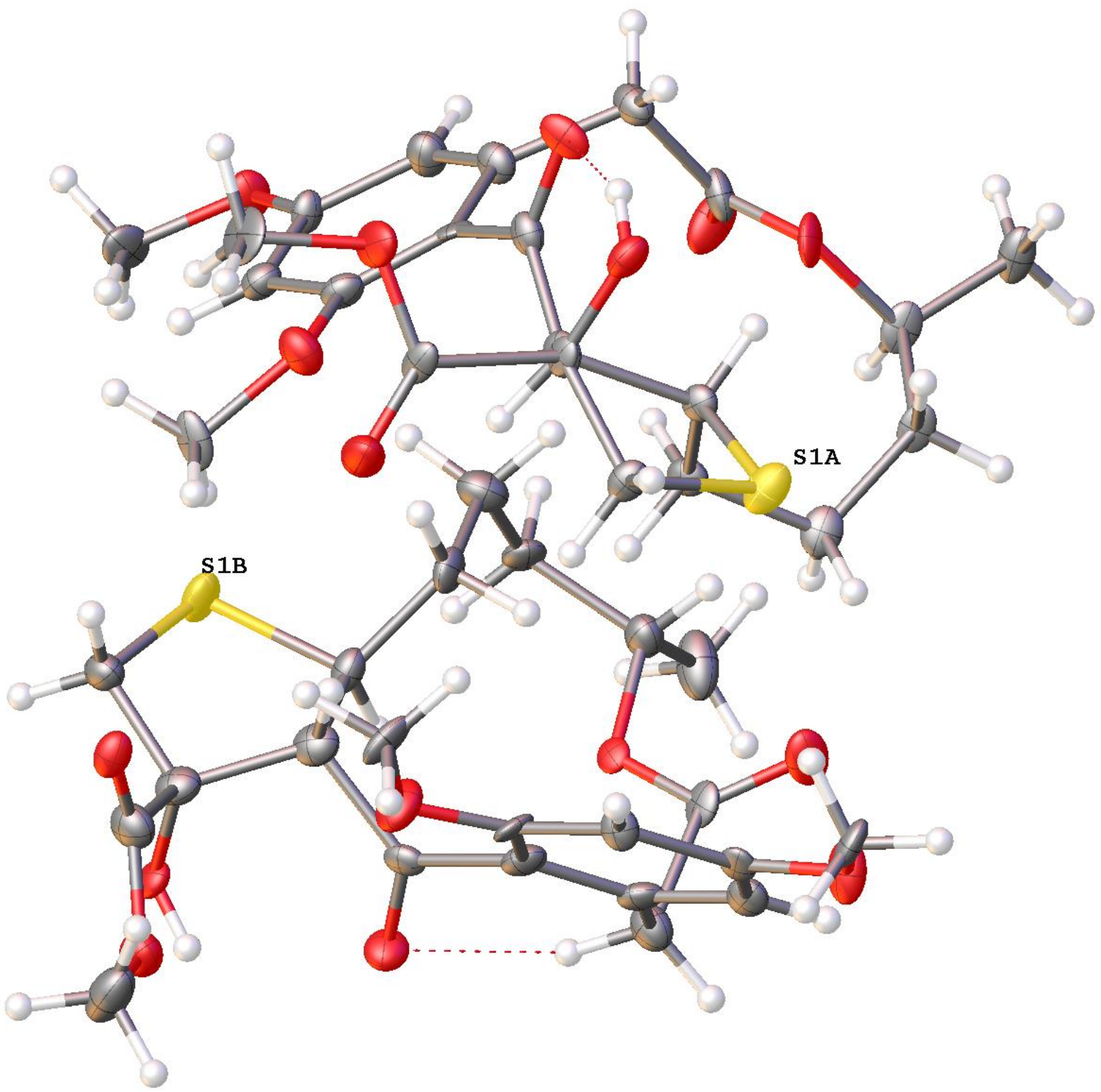

Figure S5. ORTEP-style image of both molecules in the asymmetric unit. 
2.1 Experimental details of dimethoxymethyl ester of cyclothiocurvularin B (9) X-Ray diffraction analysis

A. Dimethoxymethyl ester of cyclothiocurvularin B (9) Crystal Data

Empirical Formula

Formula Weight

Crystal Colour, Habit

Crystal Dimensions

Crystal System

Lattice Type

Lattice Parameters

Space Group

$\mathrm{Z}$ value

Dcalc

F000

$\mu($ Mo-K $\alpha)$
$\mathrm{C}_{22} \mathrm{H}_{28} \mathrm{O}_{8} \mathrm{~S}$

452.50

colourless, plate

$0.12 \times 0.27 \times 0.37 \mathrm{~mm}$

tetragonal

Primitive

$a=10.019(3) \AA$

$\mathrm{b}=10.019(3) \AA$

$c=42.875(12) \AA$

$\alpha=90^{\circ}$

$\beta=90^{\circ}$

$\gamma=90^{\circ}$

$\mathrm{V}=4304(3) \AA^{3}$

$P_{4}$ (\#29)

4

$1.397 \mathrm{~g} / \mathrm{cm}^{3}$

1920.00

$1.97 \mathrm{~cm}^{-1}$ 
B. Intensity Measurements

Diffractometer

Radiation

Data Images

Detector Position

$2 \theta_{\max }$

No. of Reflections Measured

Corrections

C. Structure Solution and Refinement

Structure Solution

Refinement

Function Minimized

Least Squares Weights

Anomalous Dispersion

No. Observations $(\mathrm{I}>0.00 \sigma(\mathrm{I}))$

No. Variables

Reflection/Parameter Ratio

Residuals (refined on $\mathrm{F}^{2}$, all data): R1; wR2

Goodness of Fit Indicator

No. Observations $(\mathrm{I}>2.00 \sigma(\mathrm{I}))$

Residuals (refined on $\mathrm{F}^{2}$ ): R1; wR2

Max Shift/Error in Final Cycle
Bruker APEX DUO

$\operatorname{Mo-K} \alpha(\lambda=0.71073 \AA)$

1696 exposures@10.0 seconds

$59.53 \mathrm{~mm}$

$48.6^{\circ}$

Total: 26253

Unique: $6912\left(\mathrm{R}_{\text {int }}=0.098\right)$

Absorption $\left(\mathrm{T}_{\min }=0.747, \mathrm{~T}_{\max }=0.994\right)$

Lorentz-polarization

Direct Methods (XT)

Full-matrix least-squares on $\mathrm{F}^{2}$

$\sum \mathrm{w}\left(\mathrm{Fo}^{2}-\mathrm{Fc}^{2}\right)^{2}$

$\mathrm{w}=1 /\left(\sigma^{2}\left(\mathrm{Fo}^{2}\right)+(0.1530 \mathrm{P})^{2}+34.0040 \mathrm{P}\right)$

All non-hydrogen atoms

6912

570

12.13

$0.117 ; 0.304$

1.05

6318

$0.112 ; 0.300$

0.00 
Maximum peak in Final Diff. Map

Minimum peak in Final Diff. Map
$0.57 \mathrm{e}^{-/} \AA^{3}$

$-0.60 \mathrm{e}^{-} / \AA^{3}$

Table S9. Fractional Atomic Coordinates $\left(\times 10^{4}\right)$ and Equivalent Isotropic Displacement Parameters $\left(\AA^{2} \times 10^{3}\right)$ for dimethoxymethyl ester of cyclothiocurvularin $B(9)$. $U_{\text {eq }}$ is defined as $1 / 3$ of of the trace of the orthogonalised $\mathrm{U}_{\mathrm{IJ}}$ tensor.

\begin{tabular}{|c|c|c|c|c|}
\hline Atom & $x$ & $y$ & $z$ & $\mathbf{U}(\mathbf{e q})$ \\
\hline S1A & $6048(5)$ & $7361(5)$ & $1000.8(12)$ & $26.4(11)$ \\
\hline O1A & $6622(14)$ & $1064(16)$ & $618(3)$ & $35(4)$ \\
\hline $\mathrm{O} 2 \mathrm{~A}$ & $11115(14)$ & $1006(13)$ & $151(3)$ & $25(3)$ \\
\hline $\mathrm{O} 3 \mathrm{~A}$ & $10762(15)$ & $5508(14)$ & $537(3)$ & $29(3)$ \\
\hline $\mathrm{O} 4 \mathrm{~A}$ & $9184(13)$ & $4494(12)$ & $1274(3)$ & $21(3)$ \\
\hline $\mathrm{O5A}$ & $5917(13)$ & $2673(14)$ & $949(3)$ & $23(3)$ \\
\hline O6A & 8487 (13) & $6986(13)$ & $1435(3)$ & $22(3)$ \\
\hline O7A & $10267(15)$ & $8487(16)$ & $817(4)$ & $41(4)$ \\
\hline O8A & $11006(14)$ & $7193(14)$ & $1221(3)$ & $29(3)$ \\
\hline $\mathrm{C} 1 \mathrm{~A}$ & $6828(18)$ & $1861(19)$ & $833(4)$ & $19(3)$ \\
\hline $\mathrm{C} 2 \mathrm{~A}$ & 8155 (18) & $1922(18)$ & $1002(4)$ & $18(3)$ \\
\hline $\mathrm{C} 3 \mathrm{~A}$ & $9264(18)$ & $2452(18)$ & $772(4)$ & $17(3)$ \\
\hline $\mathrm{C} 4 \mathrm{~A}$ & 9783 (19) & $1568(19)$ & $567(4)$ & $21(3)$ \\
\hline $\mathrm{C} 5 \mathrm{~A}$ & $10727(18)$ & $2016(18)$ & $344(4)$ & $18(3)$ \\
\hline C6A & 11044 (19) & $3325(18)$ & $317(4)$ & $19(3)$ \\
\hline C7A & $10423(19)$ & $4246(19)$ & $535(4)$ & $20(3)$ \\
\hline $\mathrm{C} 8 \mathrm{~A}$ & $9604(17)$ & $3781(17)$ & $762(4)$ & $13(3)$ \\
\hline
\end{tabular}




\begin{tabular}{|c|c|c|c|c|}
\hline C9A & $9092(18)$ & $4720(18)$ & $997(4)$ & $19(3)$ \\
\hline C10A & $8380(19)$ & $5989(19)$ & $904(4)$ & $23(3)$ \\
\hline C11A & $6781(17)$ & $5813(18)$ & $899(4)$ & $16(3)$ \\
\hline C12A & $6414(18)$ & $5272(18)$ & $582(4)$ & $18(3)$ \\
\hline C13A & $4792(19)$ & $5080(20)$ & $556(4)$ & $22(4)$ \\
\hline C14A & $4275(18)$ & $4202(17)$ & $801(4)$ & $18(3)$ \\
\hline $\mathrm{C} 15 \mathrm{~A}$ & $4628(19)$ & $2710(20)$ & $816(5)$ & $23(3)$ \\
\hline C16A & $3730(20)$ & $1876(19)$ & $1004(5)$ & $27(4)$ \\
\hline C17A & $7585(17)$ & $8297(17)$ & $1017(4)$ & $19(3)$ \\
\hline C18A & $8634(18)$ & $7262(18)$ & $1121(4)$ & $17(3)$ \\
\hline C19A & $10058(18)$ & $7792(18)$ & $1033(4)$ & $18(3)$ \\
\hline C20A & $12110(20)$ & $1410(20)$ & $-71(5)$ & $26(4)$ \\
\hline $\mathrm{C} 21 \mathrm{~A}$ & $11470(20)$ & $6097(19)$ & $272(5)$ & $24(4)$ \\
\hline $\mathrm{C} 22 \mathrm{~A}$ & $12340(20)$ & $7490(30)$ & $1144(5)$ & $37(5)$ \\
\hline S1B & $10038(5)$ & $3633(5)$ & $-597.7(13)$ & $29.1(11)$ \\
\hline O1B & $3557(14)$ & $4103(13)$ & $-277(3)$ & $29(3)$ \\
\hline $\mathrm{O} 2 \mathrm{~B}$ & 3551 (14) & $8589(14)$ & $214(3)$ & $29(3)$ \\
\hline O3B & $8042(14)$ & $8299(16)$ & $-164(3)$ & $32(3)$ \\
\hline O4B & $7064(13)$ & $6521(13)$ & $-892(3)$ & $23(3)$ \\
\hline O5B & $5294(11)$ & $3591(12)$ & $-581(3)$ & $14(2)$ \\
\hline O6B & $9522(12)$ & $6050(13)$ & $-1039(3)$ & $19(3)$ \\
\hline O7B & $10949(12)$ & $7877(12)$ & $-420(3)$ & $18(3)$ \\
\hline O8B & $9787(16)$ & $8540(15)$ & $-840(3)$ & $36(3)$ \\
\hline C1B & $4360(19)$ & $4382(19)$ & $-486(4)$ & $20(3)$ \\
\hline
\end{tabular}




\begin{tabular}{|c|c|c|c|c|}
\hline $\mathrm{C} 2 \mathrm{~B}$ & $4530(20)$ & $5830(20)$ & $-648(5)$ & $26(4)$ \\
\hline $\mathrm{C} 3 \mathrm{~B}$ & $4980(20)$ & $6718(19)$ & $-414(4)$ & $23(3)$ \\
\hline $\mathrm{C} 4 \mathrm{~B}$ & $4070(20)$ & $7240(20)$ & $-202(4)$ & $25(4)$ \\
\hline C5B & $4513(19)$ & $8109(18)$ & $28(4)$ & $18(3)$ \\
\hline C6B & $5890(20)$ & $8520(20)$ & $57(4)$ & $22(3)$ \\
\hline $\mathrm{C} 7 \mathrm{~B}$ & $6795(18)$ & $8004(18)$ & $-148(4)$ & $16(3)$ \\
\hline $\mathrm{C} 8 \mathrm{~B}$ & $6330(20)$ & 7068 (19) & $-382(4)$ & $22(3)$ \\
\hline C9B & $7353(18)$ & $6501(17)$ & $-615(4)$ & $16(3)$ \\
\hline C10B & $8620(20)$ & $5837(19)$ & $-506(4)$ & $23(3)$ \\
\hline C11B & $8397(18)$ & $4371(18)$ & $-512(4)$ & $18(3)$ \\
\hline C12B & $7869(18)$ & $3773(18)$ & $-207(4)$ & $18(3)$ \\
\hline C13B & $7670(20)$ & $2370(20)$ & $-190(5)$ & $28(4)$ \\
\hline C14B & $6780(20)$ & $1710(20)$ & $-433(5)$ & $24(4)$ \\
\hline C15B & $5340(20)$ & $2210(20)$ & $-446(5)$ & $27(4)$ \\
\hline C16B & $4460(20)$ & $1220(20)$ & $-638(5)$ & $33(5)$ \\
\hline C17B & $10878(19)$ & $5234(19)$ & $-649(4)$ & $23(4)$ \\
\hline C18B & $9790(20)$ & $6150(20)$ & $-722(4)$ & $24(3)$ \\
\hline C19B & $10320(20)$ & $7670(20)$ & $-637(5)$ & $27(4)$ \\
\hline C20B & $3834(17)$ & 9522 (19) & $461(4)$ & $20(4)$ \\
\hline C21B & $8648(19)$ & $8887(19)$ & $118(5)$ & $24(4)$ \\
\hline $\mathrm{C} 22 \mathrm{~B}$ & $10150(20)$ & $9890(20)$ & $-745(6)$ & $43(5)$ \\
\hline
\end{tabular}


Table S10. Anisotropic Displacement Parameters $\left(\AA^{2} \times 10^{3}\right)$ for dimethoxymethyl ester of cyclothiocurvularin B (9). The Anisotropic displacement factor exponent takes the form: $2 \pi^{2}\left[h^{2} a^{* 2} U_{11}+2 h k a * b * U_{12}+\ldots\right]$.

\begin{tabular}{|c|c|c|c|c|c|c|}
\hline Atom & $\mathbf{U}_{11}$ & $\mathbf{U}_{22}$ & $\mathbf{U}_{33}$ & $\mathbf{U}_{23}$ & $\mathbf{U}_{13}$ & $\mathbf{U}_{12}$ \\
\hline S1A & $17(2)$ & $17(2)$ & $45(3)$ & $-5(2)$ & $-4(2)$ & $-2.8(18)$ \\
\hline O1A & $24(7)$ & $35(7)$ & $46(7)$ & $-21(6)$ & $-7(6)$ & $11(6)$ \\
\hline $\mathrm{O} 2 \mathrm{~A}$ & $23(5)$ & $21(5)$ & $31(5)$ & $-2(4)$ & $6(4)$ & $2(4)$ \\
\hline $\mathrm{O} 3 \mathrm{~A}$ & $30(6)$ & $25(5)$ & $31(5)$ & $4(4)$ & $3(4)$ & $-4(4)$ \\
\hline $\mathrm{O} 4 \mathrm{~A}$ & $28(7)$ & $12(6)$ & $23(5)$ & $-2(4)$ & $-3(5)$ & $-13(5)$ \\
\hline O5A & $17(4)$ & $29(5)$ & $23(5)$ & $-3(4)$ & $6(4)$ & $-1(4)$ \\
\hline O6A & $16(6)$ & $20(7)$ & $30(5)$ & $0(4)$ & $-2(4)$ & $6(5)$ \\
\hline O7A & $26(7)$ & $45(9)$ & $51(7)$ & $30(7)$ & $3(6)$ & $4(6)$ \\
\hline O8A & $23(5)$ & $24(5)$ & $40(5)$ & $8(4)$ & $-4(4)$ & $-5(4)$ \\
\hline $\mathrm{C} 1 \mathrm{~A}$ & $18(5)$ & $18(6)$ & $22(5)$ & $-1(4)$ & $3(4)$ & $-9(4)$ \\
\hline $\mathrm{C} 2 \mathrm{~A}$ & $17(5)$ & $19(6)$ & $18(5)$ & $2(5)$ & $-3(4)$ & $-6(4)$ \\
\hline C3A & $17(5)$ & $16(5)$ & $18(5)$ & $1(4)$ & $-1(4)$ & $-3(4)$ \\
\hline $\mathrm{C} 4 \mathrm{~A}$ & $20(6)$ & $18(5)$ & $25(5)$ & $2(4)$ & $2(5)$ & $1(4)$ \\
\hline $\mathrm{C} 5 \mathrm{~A}$ & $17(6)$ & $17(5)$ & $19(5)$ & $-2(4)$ & $1(4)$ & $-2(4)$ \\
\hline C6A & $17(6)$ & $18(5)$ & $23(6)$ & $1(4)$ & $-1(5)$ & $2(4)$ \\
\hline C7A & $19(6)$ & $18(5)$ & $22(5)$ & $0(4)$ & $-3(4)$ & $1(4)$ \\
\hline C8A & $9(5)$ & $12(4)$ & $17(5)$ & $4(4)$ & $-9(4)$ & $4(4)$ \\
\hline C9A & $16(6)$ & $19(5)$ & $21(5)$ & $-2(4)$ & $1(4)$ & $-3(4)$ \\
\hline $\mathrm{C} 10 \mathrm{~A}$ & $24(5)$ & $20(5)$ & $25(6)$ & $-2(4)$ & $-1(4)$ & $-3(4)$ \\
\hline C11A & $16(5)$ & $13(5)$ & $18(5)$ & $-1(4)$ & $-2(4)$ & $-4(4)$ \\
\hline
\end{tabular}




\begin{tabular}{|c|c|c|c|c|c|c|}
\hline $\mathrm{C} 12 \mathrm{~A}$ & $20(5)$ & $12(6)$ & $21(5)$ & $0(5)$ & $-2(4)$ & $-3(5)$ \\
\hline C13A & $21(6)$ & $20(6)$ & $24(6)$ & $-6(5)$ & $-1(5)$ & $0(5)$ \\
\hline C14A & $16(6)$ & $11(5)$ & $25(6)$ & $-5(4)$ & $-3(5)$ & $-2(4)$ \\
\hline C15A & $20(5)$ & $19(5)$ & $29(6)$ & $-6(5)$ & $-1(5)$ & $2(4)$ \\
\hline C16A & $19(8)$ & $14(8)$ & $48(10)$ & $-5(7)$ & $5(7)$ & $-2(6)$ \\
\hline C17A & $16(5)$ & $10(5)$ & $29(6)$ & $-2(4)$ & $-2(5)$ & $-3(4)$ \\
\hline C18A & $14(5)$ & $16(5)$ & $22(5)$ & $0(4)$ & $-1(4)$ & $-3(4)$ \\
\hline C19A & $16(5)$ & $12(5)$ & $27(5)$ & $1(4)$ & $-2(4)$ & $0(4)$ \\
\hline $\mathrm{C} 20 \mathrm{~A}$ & $27(9)$ & $20(9)$ & $32(8)$ & $4(7)$ & $6(7)$ & $17(7)$ \\
\hline C21A & $26(9)$ & $12(8)$ & $35(8)$ & $-5(7)$ & $12(7)$ & $-10(7)$ \\
\hline C22A & $18(7)$ & $44(12)$ & $48(11)$ & $-1(10)$ & $-10(7)$ & $-11(7)$ \\
\hline S1B & $15(2)$ & $25(2)$ & $47(3)$ & $5(2)$ & $6(2)$ & $9.8(19)$ \\
\hline O1B & $31(7)$ & $14(6)$ & $42(7)$ & $-2(5)$ & $12(6)$ & $8(5)$ \\
\hline $\mathrm{O} 2 \mathrm{~B}$ & $26(5)$ & $26(5)$ & $36(5)$ & $-9(4)$ & $5(4)$ & $-5(4)$ \\
\hline $\mathrm{O} 3 \mathrm{~B}$ & $23(5)$ & $38(6)$ & $36(5)$ & $-1(5)$ & $-5(4)$ & $1(4)$ \\
\hline O4B & $25(7)$ & $27(7)$ & $18(5)$ & $0(5)$ & $-5(4)$ & $-2(6)$ \\
\hline O5B & $9(4)$ & $18(4)$ & $16(4)$ & $-3(3)$ & $-6(4)$ & $-6(3)$ \\
\hline O6B & $9(6)$ & $22(7)$ & $25(5)$ & $1(4)$ & $-3(4)$ & $-1(5)$ \\
\hline O7B & $11(6)$ & $10(6)$ & $34(6)$ & $0(4)$ & $3(4)$ & $1(4)$ \\
\hline O8B & $36(6)$ & $33(5)$ & $38(5)$ & $8(4)$ & $-2(5)$ & $3(4)$ \\
\hline C1B & $15(5)$ & $20(5)$ & $26(6)$ & $-4(4)$ & $-3(4)$ & $-5(4)$ \\
\hline $\mathrm{C} 2 \mathrm{~B}$ & $27(6)$ & $26(5)$ & $26(6)$ & $0(4)$ & $2(5)$ & $-4(5)$ \\
\hline $\mathrm{C} 3 \mathrm{~B}$ & $25(5)$ & $19(6)$ & $25(5)$ & $2(4)$ & $-2(4)$ & $3(4)$ \\
\hline C4B & $25(6)$ & $23(6)$ & $28(5)$ & $0(5)$ & $1(4)$ & $1(5)$ \\
\hline
\end{tabular}




\begin{tabular}{|c|c|c|c|c|c|c|}
\hline C5B & $19(5)$ & $13(6)$ & $22(5)$ & $6(4)$ & $2(4)$ & $0(4)$ \\
\hline C6B & $22(5)$ & $20(6)$ & $23(6)$ & $-1(5)$ & $2(4)$ & $-1(4)$ \\
\hline C7B & $16(5)$ & $15(6)$ & $18(5)$ & $2(4)$ & $-2(4)$ & $8(4)$ \\
\hline $\mathrm{C} 8 \mathrm{~B}$ & $22(5)$ & $17(6)$ & $25(5)$ & $4(4)$ & $-3(4)$ & $4(4)$ \\
\hline C9B & $17(5)$ & $13(5)$ & $17(5)$ & $3(4)$ & $-3(4)$ & $-3(4)$ \\
\hline C10B & $21(5)$ & $23(5)$ & $24(5)$ & $1(4)$ & $-3(4)$ & $-2(4)$ \\
\hline C11B & $14(5)$ & $19(5)$ & $21(6)$ & $-2(4)$ & $1(4)$ & $2(4)$ \\
\hline C12B & $13(6)$ & $23(5)$ & $18(5)$ & $-4(4)$ & $3(5)$ & $4(4)$ \\
\hline C13B & $31(7)$ & $26(6)$ & $26(6)$ & $4(5)$ & $1(5)$ & $0(5)$ \\
\hline C14B & $22(6)$ & $24(6)$ & $27(6)$ & $5(5)$ & $-1(5)$ & $4(5)$ \\
\hline C15B & $24(6)$ & $26(6)$ & $30(6)$ & $2(5)$ & $2(5)$ & $-1(4)$ \\
\hline C16B & $25(8)$ & $45(10)$ & $28(9)$ & $-12(8)$ & $5(7)$ & $-10(8)$ \\
\hline C17B & $20(5)$ & $23(6)$ & $25(6)$ & $5(5)$ & $0(5)$ & $-3(4)$ \\
\hline C18B & $22(5)$ & $24(5)$ & $25(5)$ & $1(4)$ & $-4(4)$ & $-2(4)$ \\
\hline C19B & $26(6)$ & $24(5)$ & $30(6)$ & $3(4)$ & $0(5)$ & $-4(4)$ \\
\hline $\mathrm{C} 20 \mathrm{~B}$ & $7(8)$ & $21(8)$ & $30(7)$ & $-4(6)$ & $-1(6)$ & $4(7)$ \\
\hline $\mathrm{C} 21 \mathrm{~B}$ & $17(8)$ & $14(8)$ & $41(9)$ & $-7(7)$ & $-9(7)$ & $15(7)$ \\
\hline C22B & 33 (12) & $29(8)$ & $67(14)$ & $7(7)$ & $0(10)$ & $6(7)$ \\
\hline
\end{tabular}


Table S11. Bond Lengths for dimethoxymethyl ester of cyclothiocurvularin B (9).

\begin{tabular}{|c|c|c|c|c|c|}
\hline \multicolumn{2}{|c|}{ Atom Atom } & \multirow{2}{*}{$\begin{array}{r}\text { Length/Å } \\
1.771(18)\end{array}$} & \multicolumn{2}{|c|}{ Atom Atom } & \multirow{2}{*}{$\begin{array}{r}\text { Length/A } \\
1.840(18)\end{array}$} \\
\hline S1A & C11A & & S1B & $\mathrm{C} 11 \mathrm{~B}$ & \\
\hline S1A & C17A & $1.805(18)$ & S1B & C17B & $1.825(19)$ \\
\hline O1A & $\mathrm{C} 1 \mathrm{~A}$ & $1.24(2)$ & O1B & $\mathrm{C} 1 \mathrm{~B}$ & $1.24(2)$ \\
\hline $\mathrm{O} 2 \mathrm{~A}$ & $\mathrm{C} 5 \mathrm{~A}$ & $1.36(2)$ & $\mathrm{O} 2 \mathrm{~B}$ & C5B & $1.34(2)$ \\
\hline $\mathrm{O} 2 \mathrm{~A}$ & C20A & $1.43(2)$ & $\mathrm{O} 2 \mathrm{~B}$ & C20B & $1.44(2)$ \\
\hline $\mathrm{O} 3 \mathrm{~A}$ & C7A & $1.31(2)$ & $\mathrm{O} 3 \mathrm{~B}$ & $\mathrm{C} 7 \mathrm{~B}$ & $1.29(2)$ \\
\hline $\mathrm{O} 3 \mathrm{~A}$ & $\mathrm{C} 21 \mathrm{~A}$ & $1.46(2)$ & $\mathrm{O} 3 \mathrm{~B}$ & $\mathrm{C} 21 \mathrm{~B}$ & $1.47(2)$ \\
\hline $\mathrm{O} 4 \mathrm{~A}$ & C9A & $1.21(2)$ & O4B & $\mathrm{C} 9 \mathrm{~B}$ & $1.22(2)$ \\
\hline O5A & $\mathrm{C} 1 \mathrm{~A}$ & $1.32(2)$ & $\mathrm{O} 5 \mathrm{~B}$ & $\mathrm{C} 1 \mathrm{~B}$ & $1.29(2)$ \\
\hline O5A & $\mathrm{C} 15 \mathrm{~A}$ & $1.41(2)$ & O5B & $\mathrm{C} 15 \mathrm{~B}$ & $1.51(2)$ \\
\hline O6A & C18A & $1.38(2)$ & O6B & $\mathrm{C} 18 \mathrm{~B}$ & $1.39(2)$ \\
\hline O7A & C19A & $1.18(2)$ & O7B & C19B & $1.14(2)$ \\
\hline O8A & C19A & $1.38(2)$ & O8B & C19B & $1.34(3)$ \\
\hline O8A & C22A & $1.40(3)$ & O8B & $\mathrm{C} 22 \mathrm{~B}$ & $1.46(3)$ \\
\hline C1A & $\mathrm{C} 2 \mathrm{~A}$ & $1.51(3)$ & C1B & $\mathrm{C} 2 \mathrm{~B}$ & $1.62(3)$ \\
\hline $\mathrm{C} 2 \mathrm{~A}$ & $\mathrm{C} 3 \mathrm{~A}$ & $1.58(2)$ & $\mathrm{C} 2 \mathrm{~B}$ & $\mathrm{C} 3 \mathrm{~B}$ & $1.41(3)$ \\
\hline $\mathrm{C} 3 \mathrm{~A}$ & $\mathrm{C} 4 \mathrm{~A}$ & $1.35(3)$ & $\mathrm{C} 3 \mathrm{~B}$ & $\mathrm{C} 4 \mathrm{~B}$ & $1.39(3)$ \\
\hline $\mathrm{C} 3 \mathrm{~A}$ & $\mathrm{C} 8 \mathrm{~A}$ & $1.37(3)$ & $\mathrm{C} 3 \mathrm{~B}$ & $\mathrm{C} 8 \mathrm{~B}$ & $1.40(3)$ \\
\hline $\mathrm{C} 4 \mathrm{~A}$ & $\mathrm{C} 5 \mathrm{~A}$ & $1.42(3)$ & $\mathrm{C} 4 \mathrm{~B}$ & C5B & $1.39(3)$ \\
\hline $\mathrm{C} 5 \mathrm{~A}$ & C6A & $1.35(3)$ & $\mathrm{C} 5 \mathrm{~B}$ & C6B & $1.45(3)$ \\
\hline C6A & C7A & $1.45(3)$ & C6B & C7B & $1.36(3)$ \\
\hline
\end{tabular}




$\begin{array}{llll}\text { C7A C8A } & 1.36(3) \text { C7B C8B } & 1.45(3) \\ \text { C8A C9A } & 1.47(3) \text { C8B C9B } & 1.54(3) \\ \text { C9A C10A } & 1.51(3) \text { C9B C10B } & 1.51(3) \\ \text { C10AC11A } & 1.61(3) \text { C10B C11B } & 1.49(3) \\ \text { C10AC18A } & 1.60(3) \text { C10B C18B } & 1.52(3) \\ \text { C11AC12A } & 1.51(2) \text { C11B C12B } & 1.53(2) \\ \text { C12AC13A } & 1.64(2) \text { C12B C13B } & 1.43(3) \\ \text { C13AC14A } & 1.46(3) \text { C13B C14B } & 1.52(3) \\ \text { C14AC15A } & 1.54(3) \text { C14B C15B } & 1.53(3) \\ \text { C15AC16A } & 1.47(3) & \text { C15B C16B } & 1.56(3) \\ \text { C17AC18A } & 1.54(3) & \text { C17B C18B } & 1.46(3) \\ \text { C18AC19A } & 1.57(3) & \text { C18B C19B } & 1.65(3)\end{array}$

Table S12. Bond Angles for dimethoxymethyl ester of cyclothiocurvularin B (9).

\begin{tabular}{|c|c|c|c|c|}
\hline Atom Atom Atom & Angle $/^{\circ}$ & Atom Atom & Atom & Angle $/^{\circ}$ \\
\hline C11AS1A C17A & $96.3(8)$ & C17B S1B & $\mathrm{C} 11 \mathrm{~B}$ & $94.8(9)$ \\
\hline C5A O2A C20A & $112.9(14)$ & $\mathrm{C} 5 \mathrm{~B} \quad \mathrm{O} 2 \mathrm{~B}$ & C20B & $121.9(15)$ \\
\hline C7A O3A C21A & $120.6(15)$ & $\mathrm{C} 7 \mathrm{~B} \quad \mathrm{O} 3 \mathrm{~B}$ & $\mathrm{C} 21 \mathrm{~B}$ & $116.7(15)$ \\
\hline $\mathrm{C} 1 \mathrm{~A} \quad \mathrm{O} 5 \mathrm{~A} \quad \mathrm{C} 15 \mathrm{~A}$ & $119.8(14)$ & $\mathrm{C} 1 \mathrm{~B} \quad \mathrm{O5B}$ & $\mathrm{C} 15 \mathrm{~B}$ & $117.9(14)$ \\
\hline C19A O8A C22A & $115.0(15)$ & C19B O8B & $\mathrm{C} 22 \mathrm{~B}$ & $108.9(17)$ \\
\hline O1A C1A O5A & $124.3(17)$ & O1B C1B & O5B & $124.1(18)$ \\
\hline $\mathrm{O} 1 \mathrm{~A} \quad \mathrm{C} 1 \mathrm{~A} \quad \mathrm{C} 2 \mathrm{~A}$ & $121.9(17)$ & O1B C1B & $\mathrm{C} 2 \mathrm{~B}$ & $125.7(17)$ \\
\hline O5A C1A C2A & $113.7(15)$ & O5B C1B & $\mathrm{C} 2 \mathrm{~B}$ & $109.7(16)$ \\
\hline C1A C2A C3A & $109.5(14)$ & $\mathrm{C} 3 \mathrm{~B} \quad \mathrm{C} 2 \mathrm{~B}$ & $\mathrm{C} 1 \mathrm{~B}$ & $107.1(16)$ \\
\hline
\end{tabular}


C4A C3A C2A

C4A C3A C8A

C8A C3A C2A

C3A C4A C5A

O2A C5A C4A

C6A C5A O2A

C6A C5A C4A

C5A C6A C7A

O3A C7A C6A

O3A C7A C8A

C8A C7A C6A

C3A C8A C9A

C7A C8A C3A

C7A C8A C9A

O4A C9A C8A

O4A C9A C10A

C8A C9A C10A

C9A C10AC11A

C9A C10AC18A

C18A C10A C11A

C10A C11AS1A

C12A C11AS1A

C12A C11A C10A

C11A C12AC13A
117.2(16) C4B C3B C2B

121.3(17) C4B C3B C8B

121.3(16) C8B C3B C2B

119.2(18) C5B C4B C3B

111.4(16) O2B C5B C4B

126.8(17) O2B C5B C6B

121.4(17) C4B C5B C6B

117.4(17) C7B C6B C5B

$120.5(17)$ O3B C7B C6B

118.9(17) O3B C7B C8B

120.2(17) C6B C7B C8B

120.8(16) C3B C8B C7B

120.3(17) C3B C8B C9B

118.9(16) C7B C8B C9B

$121.6(16)$ O4B C9B C8B

$116.8(16)$ O4B C9B C10B

121.5 (15) C10B C9B C8B

112.3 (15) C9B C10B C18B

116.3 (15) C11B C10B C9B

104.7 (14) C11B C10B C18B

108.3 (12) C10B C11B S1B

115.7 (13) C10B C11B C12B

107.1 (15) C12B C11B S1B

110.1 (14) C13B C12B C11B
$119.3(19)$

$118.2(19)$

$122.5(19)$

$119.4(19)$

$114.7(17)$

$122.2(17)$

$123.0(17)$

$118.0(17)$

$126.2(17)$

$115.3(16)$

$118.5(17)$

$122.9(18)$

$119.0(17)$

$118.0(16)$

$117.7(15)$

$120.4(16)$

$121.6(15)$

$111.5(15)$

$107.8(16)$

$108.2(16)$

105.4(13)

$115.2(15)$

$108.8(12)$

$118.5(16)$ 


\begin{tabular}{|c|c|c|c|}
\hline C14AC13AC12A & $111.8(15)$ & C12B C13B C14B & $118.6(17)$ \\
\hline C13A C14AC15A & $122.3(16)$ & C13B C14B C15B & $115.6(17)$ \\
\hline O5A C15AC14A & $104.7(15)$ & O5B C15B C14B & $110.2(15)$ \\
\hline O5A C15AC16A & $108.8(16)$ & O5B C15B C16B & $111.2(16)$ \\
\hline C16A C15AC14A & $115.9(15)$ & C14B C15B C16B & $110.4(17)$ \\
\hline C18AC17AS1A & $104.1(11)$ & C18B C17B S1B & $103.6(13)$ \\
\hline O6A C18AC10A & $113.0(15)$ & O6B C18B C10B & $115.6(15)$ \\
\hline O6A C18AC17A & $110.2(15)$ & O6B C18B C17B & $107.7(16)$ \\
\hline O6A C18AC19A & $113.5(14)$ & O6B C18B C19B & $110.3(15)$ \\
\hline C17A C18AC10A & $105.0(14)$ & C10B C18B C19B & $107.8(15)$ \\
\hline C17A C18AC19A & $108.8(14)$ & C17B C18B C10B & $108.2(16)$ \\
\hline C19A C18AC10A & $105.9(14)$ & C17B C18B C19B & $106.8(15)$ \\
\hline O7A C19AO8A & $126.3(18)$ & O7B C19B O8B & $129(2)$ \\
\hline O7A C19AC18A & $123.5(17)$ & O7B C19B C18B & $121.9(17)$ \\
\hline O8A C19AC18A & $109.8(14)$ & O8B C19B C18B & $109.0(16)$ \\
\hline
\end{tabular}

Table S13. Hydrogen Bonds for dimethoxymethyl ester of cyclothiocurvularin B (9).

$\begin{array}{rrrrrrr}\mathbf{D} & \mathbf{H} & \mathbf{A} & \mathbf{d}(\mathbf{D}-\mathbf{H}) / \AA & \mathbf{d}(\mathbf{H}-\mathbf{A}) / \AA & \mathbf{d}(\mathbf{D}-\mathbf{A}) / \mathbf{A} & \mathbf{D}^{-H}-\mathbf{A}^{\circ} \\ \text { O6A } & \text { H6A } & \text { O4A } & 0.84 & 1.95 & 2.683(18) & 144.6 \\ \text { C2B } & \text { H2BA } & \text { O4B } & 0.99 & 2.04 & 2.83(2) & 134.8\end{array}$


Table S14. Torsion Angles for dimethoxymethyl ester of cyclothiocurvularin B (9).

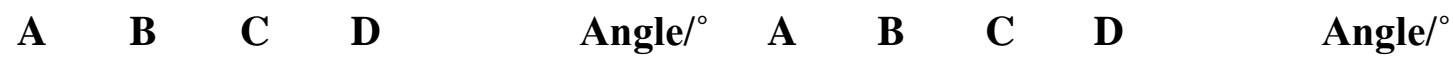
S1A C11A C12A C13A $\quad-58.4(18)$ S1B C11B C12B C13B $\quad-60(2)$ S1A C17A C18A O6A $\quad-74.6(15)$ S1B C17B C18B O6B $-83.4(15)$ S1A C17A C18A C10A $47.3(15)$ S1B C17B C18B C10B $42.2(17)$ S1A C17A C18A C19A $160.3(12)$ S1B C17B C18B C19B $158.0(12)$

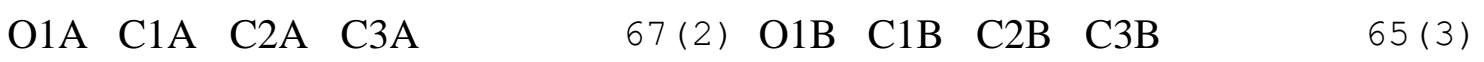
O2A C5A C6A C7A $174.8(17)$ O2B C5B C6B C7B $\quad-178.4(17)$ O3A C7A C8A C3A $\quad-178.0(16)$ O3B C7B C8B C3B $\quad-174.9(18)$ O3A C7A C8A C9A 2(3) O3B C7B C8B C9B O4A C9A C10A C11A $\quad-83(2)$ O4B C9B C10B C11B $\quad-77(2)$ O4A C9A C10A C18A $\quad 38(2)$ O4B C9B C10B C18B O5A C1A C2A C3A $\quad-116.2(16)$ O5B C1B C2B C3B $\quad-108.0(18)$ O6A C18A C19A 07A $-153.2(19)$ O6B C18B C19B O7B $-156.8(18)$ O6A C18A C19A O8A $34(2)$ O6B C18B C19B O8B 29(2) C1A O5A C15A C14A $-137.3(16)$ C1B O5B C15B C14B $-149.1(16)$ C1A O5A C15A C16A 98.2(19) C1B O5B C15B C16B 88.2(19)

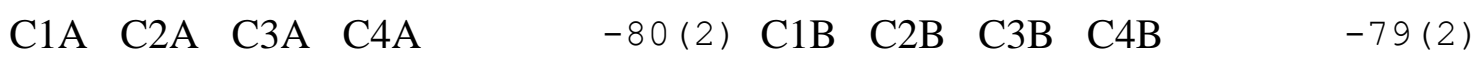

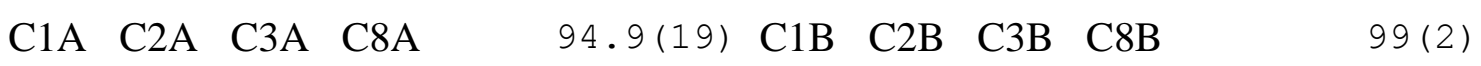
C2A C3A C4A C5A $176.4(16)$ C2B C3B C4B C5B $179.7(18)$ $\begin{array}{llllllll}\text { C2A } & \text { C3A C8A C7A } & -170.9(16) & \text { C2B C3B C8B C7B } & 178.5(18)\end{array}$

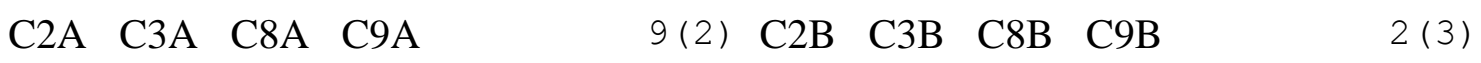




\begin{tabular}{|c|c|c|c|c|c|c|c|c|c|}
\hline C3A & $\mathrm{C} 4 \mathrm{~A}$ & C5A & $\mathrm{O} 2 \mathrm{~A}$ & $-178.3(17)$ & $\mathrm{C} 3 \mathrm{~B}$ & $\mathrm{C} 4 \mathrm{~B}$ & C5B & $\mathrm{O} 2 \mathrm{~B}$ & $178.0(17)$ \\
\hline $\mathrm{C} 3 \mathrm{~A}$ & $\mathrm{C} 4 \mathrm{~A}$ & C5A & C6A & $-5(3)$ & $\mathrm{C} 3 \mathrm{~B}$ & $\mathrm{C} 4 \mathrm{~B}$ & C5B & C6B & $1(3)$ \\
\hline $\mathrm{C} 3 \mathrm{~A}$ & $\mathrm{C} 8 \mathrm{~A}$ & C9A & $\mathrm{O} 4 \mathrm{~A}$ & $51(2)$ & C3B & $\mathrm{C} 8 \mathrm{~B}$ & $\mathrm{C} 9 \mathrm{~B}$ & $\mathrm{O} 4 \mathrm{~B}$ & $45(2)$ \\
\hline C3A & $\mathrm{C} 8 \mathrm{~A}$ & C9A & C10A & $-126.8(18)$ & $\mathrm{C} 3 \mathrm{~B}$ & $\mathrm{C} 8 \mathrm{~B}$ & C9B & $\mathrm{C} 10 \mathrm{~B}$ & $-129.8(19)$ \\
\hline $\mathrm{C} 4 \mathrm{~A}$ & $\mathrm{C} 3 \mathrm{~A}$ & C8A & C7A & $3(3)$ & $\mathrm{C} 4 \mathrm{~B}$ & $\mathrm{C} 3 \mathrm{~B}$ & $\mathrm{C} 8 \mathrm{~B}$ & $\mathrm{C} 7 \mathrm{~B}$ & $-3(3)$ \\
\hline C4A & $\mathrm{C} 3 \mathrm{~A}$ & C8A & $\mathrm{C} 9 \mathrm{~A}$ & $-176.6(17)$ & C4B & $\mathrm{C} 3 \mathrm{~B}$ & $\mathrm{C} 8 \mathrm{~B}$ & C9B & $-179.9(16)$ \\
\hline $\mathrm{C} 4 \mathrm{~A}$ & $\mathrm{C} 5 \mathrm{~A}$ & C6A & C7A & $3(3)$ & $\mathrm{C} 4 \mathrm{~B}$ & $\mathrm{C} 5 \mathrm{~B}$ & C6B & $\mathrm{C} 7 \mathrm{~B}$ & $-2(3)$ \\
\hline C5A & C6A & C7A & $\mathrm{O} 3 \mathrm{~A}$ & $174.8(17)$ & C5B & C6B & C7B & O3B & $177.1(17)$ \\
\hline C5A & C6A & C7A & $\mathrm{C} 8 \mathrm{~A}$ & $3(3)$ & C5B & C6B & $\mathrm{C} 7 \mathrm{~B}$ & $\mathrm{C} 8 \mathrm{~B}$ & $0(3)$ \\
\hline C6A & C7A & $\mathrm{C} 8 \mathrm{~A}$ & $\mathrm{C} 3 \mathrm{~A}$ & $-6(3)$ & C6B & $\mathrm{C} 7 \mathrm{~B}$ & $\mathrm{C} 8 \mathrm{~B}$ & $\mathrm{C} 3 \mathrm{~B}$ & $3(3)$ \\
\hline C6A & C7A & $\mathrm{C} 8 \mathrm{~A}$ & C9A & $174.3(16)$ & C6B & $\mathrm{C} 7 \mathrm{~B}$ & $\mathrm{C} 8 \mathrm{~B}$ & C9B & $179.3(16)$ \\
\hline C7A & $\mathrm{C} 8 \mathrm{~A}$ & C9A & $\mathrm{O} 4 \mathrm{~A}$ & $-129.0(19)$ & C7B & $\mathrm{C} 8 \mathrm{~B}$ & C9B & O4B & $-131.6(17)$ \\
\hline C7A & $\mathrm{C} 8 \mathrm{~A}$ & C9A & C10A & $53(2)$ & C7B & $\mathrm{C} 8 \mathrm{~B}$ & C9B & $\mathrm{C} 10 \mathrm{~B}$ & $53(2)$ \\
\hline $\mathrm{C} 8 \mathrm{~A}$ & $\mathrm{C} 3 \mathrm{~A}$ & $\mathrm{C} 4 \mathrm{~A}$ & $\mathrm{C} 5 \mathrm{~A}$ & $2(3)$ & $\mathrm{C} 8 \mathrm{~B}$ & $\mathrm{C} 3 \mathrm{~B}$ & C4B & C5B & $1(3)$ \\
\hline C8A & C9A & C10A & C11A & $95.0(19)$ & $\mathrm{C} 8 \mathrm{~B}$ & C9B & $\mathrm{C} 10 \mathrm{~B}$ & C11B & $98.0(19)$ \\
\hline $\mathrm{C} 8 \mathrm{~A}$ & C9A & C10A & C18A & $-144.5(17)$ & $\mathrm{C} 8 \mathrm{~B}$ & C9B & $\mathrm{C} 10 \mathrm{~B}$ & $\mathrm{C} 18 \mathrm{~B}$ & $-143.4(17)$ \\
\hline C9A & C10A & C11A & S1A & $148.5(13)$ & C9B & $\mathrm{C} 10 \mathrm{~B}$ & $\mathrm{C} 11 \mathrm{~B}$ & S1B & $149.0(12)$ \\
\hline C9A & C10A & C11A & $\mathrm{C} 12 \mathrm{~A}$ & $-86.0(18)$ & C9B & $\mathrm{C} 10 \mathrm{~B}$ & $\mathrm{C} 11 \mathrm{~B}$ & $\mathrm{C} 12 \mathrm{~B}$ & $-91.0(18)$ \\
\hline C9A & C10A & C18A & O6A & $-48(2)$ & C9B & $\mathrm{C} 10 \mathrm{~B}$ & C18B & O6B & $-45(2)$ \\
\hline C9A & C10A & C18A & C17A & $-168.5(15)$ & C9B & $\mathrm{C} 10 \mathrm{~B}$ & C18B & C17B & $-166.3(15)$ \\
\hline C9A & C10A & C18A & C19A & $76.4(19)$ & C9B & $\mathrm{C} 10 \mathrm{~B}$ & C18B & C19B & $78.5(18)$ \\
\hline C10A & $\mathrm{C} 11 \mathrm{~A}$ & $\mathrm{C} 12 \mathrm{~A}$ & C13A & $-179.2(15)$ & $\mathrm{C} 10 \mathrm{~B}$ & $\mathrm{C} 11 \mathrm{~B}$ & $\mathrm{C} 12 \mathrm{~B}$ & C13B & $-178.2(18)$ \\
\hline C10A & C18A & C19A & O7A & $82(2)$ & $\mathrm{C} 10 \mathrm{~B}$ & C18B & C19B & $\mathrm{O} 7 \mathrm{~B}$ & $76(2)$ \\
\hline C10A & C18A & C19A & O8A & $-90.6(17)$ & $\mathrm{C} 10 \mathrm{~B}$ & C18B & $\mathrm{C} 19$ & $\mathrm{O} 8 \mathrm{~B}$ & $-97.9(18)$ \\
\hline
\end{tabular}




\begin{tabular}{|c|c|c|c|c|}
\hline C11A S1A C17A C18A & $-30.9(13)$ & C11B S1B & C17B C18B & $-22.1(14)$ \\
\hline C11A C10A C18A O6A & $76.1(18)$ & C11B C10B & C18B O6B & $73(2)$ \\
\hline C11A C10A C18A C17A & $-44.0(18)$ & C11B C10B & C18B C17B & $-48(2)$ \\
\hline C11A C10A C18A C19A & $-159.0(14)$ & C11B C10B & C18B C19B & $-163.1(15)$ \\
\hline C11A C12A C13A C14A & $-57(2)$ & C11B C12B & C13B C14B & $-55(3)$ \\
\hline C12A C13A C14A C15A & $-66(2)$ & C12B C13B & C14B C15B & $-60(3)$ \\
\hline C13A C14A C15A O5A & $79(2)$ & C13B C14B & C15B O5B & $71(2)$ \\
\hline C13A C14A C15A C16A & $-161.1(18)$ & C13B C14B & C15B C16B & $-165.3(17)$ \\
\hline C15A 05A C1A O1A & $-3(3)$ & C15B O5B & C1B O1B & $5(3)$ \\
\hline C15A O5A C1A C2A & $-179.4(15)$ & C15B O5B & $\mathrm{C} 1 \mathrm{~B} \quad \mathrm{C} 2 \mathrm{~B}$ & $177.6(14)$ \\
\hline C17A S1A C11A C10A & $5.0(14)$ & C17B S1B & C11B C10B & $-3.8(13)$ \\
\hline C17A S1A C11A C12A & $-115.2(14)$ & C17B S1B & C11B C12B & $-127.9(13)$ \\
\hline C17A C18A C19A O7A & $-30(2)$ & C17B C18B & C19B O7B & $-40(2)$ \\
\hline C17A C18A C19A O8A & $157.0(15)$ & C17B C18B & C19B O8B & $146.0(17)$ \\
\hline C18A C10A C11A S1A & $21.5(17)$ & C18B C10B & C11B S1B & $28.3(17)$ \\
\hline C18A C10A C11A C12A & $146.9(15)$ & C18B C10B & C11B C12B & $148.3(16)$ \\
\hline C20A O2A C5A C4A & $-177.9(16)$ & C20B O2B & C5B C4B & $-178.7(17)$ \\
\hline C20A O2A C5A C6A & $9(3)$ & C20B O2B & C5B C6B & $-2(3)$ \\
\hline C21A O3A C7A C6A & $17(3)$ & C21B O3B & C7B C6B & $21(3)$ \\
\hline C21A O3A C7A C8A & $-170.5(17)$ & C21B O3B & C7B C8B & $-161.5(15)$ \\
\hline C22A O8A C19A O7A & $2(3)$ & C22B O8B & C19B O7B & $1(3)$ \\
\hline C22A 08A C19A C18A & $174.1(16)$ & C22B O8B & C19B C18B & $174.5(17)$ \\
\hline
\end{tabular}


Table S15. Hydrogen Atom Coordinates $\left(\AA \times 10^{4}\right)$ and Isotropic Displacement Parameters $\left(\AA^{2} \times 10^{3}\right)$ for dimethoxymethyl ester of cyclothiocurvularin $B(9)$.

Atom

$x$

8697

8399

8083

9519

11648

8672

6528

6860

6724

4355

4568

3289

4545

4670

4086

2845

3673

7812

7520

11817

12954

12226

\section{$y$}

6187

1022

2525

658

3628

6225

5137

4402

5896

5966

4704

4260

4598

2344

965

1864

2242

8669

9037

1167

970

2383 $z$

1469

1078

1184

572

162

688

1061

549

419

573

350

791

1003

600

1014

909

1216

809

1169

$-281$

$-23$

$-59$
U(eq)

33

21

21

25

23

28

19

21

21

26

26

21

21

27

41

41

41

22

22

39

39

39 


\begin{tabular}{|c|c|c|c|c|}
\hline $\mathrm{H} 21 \mathrm{~A}$ & 11238 & 5610 & 81 & 36 \\
\hline $\mathrm{H} 21 \mathrm{~B}$ & 12433 & 6041 & 308 & 36 \\
\hline $\mathrm{H} 21 \mathrm{C}$ & 11207 & 7035 & 250 & 36 \\
\hline $\mathrm{H} 22 \mathrm{~A}$ & 12514 & 7214 & 928 & 55 \\
\hline H22B & 12937 & 7014 & 1285 & 55 \\
\hline $\mathrm{H} 22 \mathrm{C}$ & 12485 & 8454 & 1164 & 55 \\
\hline H6B & 8928 & 6603 & -1088 & 28 \\
\hline $\mathrm{H} 2 \mathrm{BA}$ & 5187 & 5780 & -820 & 32 \\
\hline $\mathrm{H} 2 \mathrm{BB}$ & 3667 & 6137 & -734 & 32 \\
\hline $\mathrm{H} 4 \mathrm{~B}$ & 3153 & 7008 & -215 & 31 \\
\hline H6BA & 6160 & 9136 & 214 & 26 \\
\hline H10B & 8834 & 6132 & -289 & 27 \\
\hline H11B & 7765 & 4148 & -685 & 22 \\
\hline $\mathrm{H} 12 \mathrm{C}$ & 8498 & 4022 & -38 & 21 \\
\hline H12D & 7006 & 4210 & -159 & 21 \\
\hline $\mathrm{H} 13 \mathrm{C}$ & 7288 & 2161 & 17 & 33 \\
\hline H13D & 8555 & 1933 & -200 & 33 \\
\hline $\mathrm{H} 14 \mathrm{C}$ & 6772 & 734 & -393 & 29 \\
\hline H14D & 7195 & 1842 & -641 & 29 \\
\hline H15B & 4987 & 2241 & -228 & 32 \\
\hline H16D & 3668 & 1690 & -716 & 49 \\
\hline H16E & 4176 & 477 & -505 & 49 \\
\hline $\mathrm{H} 16 \mathrm{~F}$ & 4973 & 870 & -815 & 49 \\
\hline $\mathrm{H} 17 \mathrm{C}$ & 11346 & 5506 & -456 & \\
\hline
\end{tabular}




\begin{tabular}{|c|c|c|c|c|}
\hline H17D & 11530 & 5193 & -823 & 27 \\
\hline H20D & 4031 & 10399 & 371 & 29 \\
\hline $\mathrm{H} 20 \mathrm{E}$ & 4606 & 9210 & 580 & 29 \\
\hline $\mathrm{H} 20 \mathrm{~F}$ & 3057 & 9592 & 598 & 29 \\
\hline H21D & 8484 & 9852 & 120 & 36 \\
\hline $\mathrm{H} 21 \mathrm{E}$ & 9611 & 8720 & 117 & 36 \\
\hline $\mathrm{H} 21 \mathrm{~F}$ & 8250 & 8481 & 304 & 36 \\
\hline H22D & 11060 & 10088 & -818 & 65 \\
\hline $\mathrm{H} 22 \mathrm{E}$ & 10122 & 9958 & -517 & 65 \\
\hline $\mathrm{H} 22 \mathrm{~F}$ & 9527 & 10529 & -837 & 65 \\
\hline
\end{tabular}

